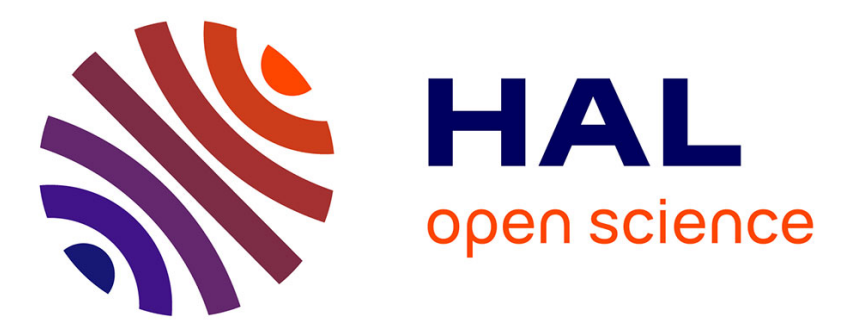

\title{
Modification de la matrice polarisation d'un faisceau lumineux lors de la traversée d'une vapeur atomique soumise au pompage optique. - Deuxième Partie
}

C. Cohen-Tannoudji, F. Laloë

\section{- To cite this version:}

C. Cohen-Tannoudji, F. Laloë. Modification de la matrice polarisation d'un faisceau lumineux lors de la traversée d'une vapeur atomique soumise au pompage optique. - Deuxième Partie. Journal de Physique, 1967, 28 (8-9), pp.722-734. 10.1051/jphys:01967002808-9072200 . jpa-00206573

\section{HAL Id: jpa-00206573 https://hal.science/jpa-00206573}

Submitted on 1 Jan 1967

HAL is a multi-disciplinary open access archive for the deposit and dissemination of scientific research documents, whether they are published or not. The documents may come from teaching and research institutions in France or abroad, or from public or private research centers.
L'archive ouverte pluridisciplinaire HAL, est destinée au dépôt et à la diffusion de documents scientifiques de niveau recherche, publiés ou non, émanant des établissements d'enseignement et de recherche français ou étrangers, des laboratoires publics ou privés. 


\title{
MODIFIGATION DE LA MATRIGE POLARISATION D'UN FAISGEAU LUMINEUX LORS DE LA TRAVERSÉE D'UNE VAPEUR ATOMIQUE SOUMISE AU POMPAGE OPTIQUE
}

\author{
Deuxième Partie $\left({ }^{1}\right)$ \\ Par G. GOHEN-TANNOUDJI et F. LALOE ( $\left.{ }^{2}\right)$, \\ Faculté des Sciences de Paris, Laboratoire de Spectroscopie Hertzienne de l'E.N.S., associé au G.N.R.S.
}

\begin{abstract}
Résumé. - Les formules générales obtenues dans un précédent article sont appliquées à un certain nombre de cas particuliers : isotopes impairs et isotopes pairs du mercure, alcalins.

Dans le cas des isotopes impairs du mercure, on souligne l'analogie étroite entre l'équation qui donne la modification de polarisation lumineuse et celle qui décrit l'évolution de la matrice densité atomique $\sigma_{\mathrm{f}}$. On étudie de façon détaillée le lien qui existe entre les propriétés de symétrie de $\sigma_{\mathrm{f}}$ et les " polarisations principales " de la vapeur. Dans le cas des isotopes pairs du mercure, on étudie l'effet d'un champ statique et d'un champ de radiofréquence sur la lumière transmise. Enfin, dans le cas des alcalins, on met en évidence des effets nouveaux liés à l'existence de " cohérences hyperfines".
\end{abstract}

Abstract. - The general formulae obtained in a previous article are applied to the particular cases of the odd and even isotopes of mercury, and of the alkali metals.

For the odd isotopes of mercury, we emphasize the close analogy between the equation giving the change of the polarization of the light and the one describing the evolution of the atomic density matrix, $\sigma_{\mathrm{f}}$. The connection between the symmetry properties of $\sigma_{\mathrm{f}}$ and the "eigen polarizations" of the vapour are worked out. For the even isotopes of mercury, we study the effect of a static and a radiofrequency magnetic field on the transmitted light. Finally, for the alkali metals, we predict some new effects due to the existence of "hyperfine coherences".

Introduction. - EFFET PARAMAGNÉTIQUe ET EFFET DiAMAGNÉTIQUE. - Dans un précédent article [1], nous avons calculé la variation $\pi_{T}-\pi_{I}$ de la matrice polarisation d'un faisceau lumineux qui traverse une vapeur atomique d'épaisseur optique faible $\left({ }^{3}\right)$ décrite dans l'état fondamental par une matrice densité $\sigma_{\mathrm{f}}(Z, t)$ quelconque. Nous avons obtenu l'équation (II.18) : $\pi_{\mathrm{T}}(t)-\pi_{\mathrm{I}}=-\left[G\left(t-Z_{0} / c\right) \pi_{I}+\pi_{\mathrm{I}} G^{+}\left(t-Z_{0} / c\right)\right]$ et donné en (II.21) et (II.22) l'expression de $G$ en fonction de $\sigma_{\mathrm{f}}(Z, t)$. Nous pouvons donc calculer le signal de détection optique le plus général sur la lumière transmise (cf. expérience ('nvisagée au § I.7) : $\Delta I=\operatorname{Tr}\left[M_{\mathrm{a}}\left(\pi_{I}-\pi_{T}\right) M_{\mathrm{a}}^{+}\right]$
$\quad=I \operatorname{Tr}\left\{M_{\mathrm{a}} G\left|\mathbf{e}_{\lambda_{0}}\right\rangle\left\langle\mathbf{e}_{\lambda_{0}}\right| M_{\mathrm{a}}^{+}\right\}+$c. c. ${ }^{4}$

(1) Cette étude fait suite à un premier article paru dans le précédent numéro du Journal de Physique [1].

(2) Attaché de Recherches au C.N.E.S.

(3) La généralisation des résultats obtenus à des épaisseurs optiques quelconques est discutée en Appendice.

(4) A partir des matrices $M_{\mathrm{p}}$ et $M_{\mathrm{a}}$ calculées au $\S$ I.6 $d$, on vérifie que $\Delta I$ est le même si l'on utilise soit un polariseur seul, soit un analyseur seul, pourvu qu'ils correspondent tous deux à la même polarisation. Ces résultats, qui apparaissent dans certains cas particuliers dans la littérature [2], sont généraux lorsque l'épaisseur optique de la cellule est faible.
La matrice $G$ joue dans notre problème un rôle fondamental; c'est une matrice $2 \times 2$ qui agit dans l'espace $\mathscr{F}$ des états de polarisation de la lumière.

Le cas le plus simple est celui où $G$ est scalaire ( $G=g 1$, où 1 est la matrice unité). On a alors : $\pi_{T}=\pi_{I}\left(1-g-g^{*}\right)$; la polarisation du faisceau transmis est la même que celle du faisceau incident; seule l'intensité totale varie dans un rapport $\left(1-g-g^{*}\right)$ indépendant de $\pi_{I}$.

C'est donc le caractère matriciel de $G$ qui est responsable de la modification de la polarisation lumineuse. En général, $G$ possède deux polarisations propres $\left|\mathbf{e}_{1}^{p}\right\rangle$ et $\left|\mathbf{e}_{2}^{p}\right\rangle$; lorsque $G$ n'est pas scalaire, ces deux « polarisations principales » sont les seules qui traversent la vapeur sans déformation. Si, par exemple, les niveaux $|\mu\rangle$ sont deux sous-niveaux issus d'un niveau de moment cinétique total $1 / 2$ et si $H_{\mathrm{e}}^{\prime} \simeq H_{\mathrm{f}}^{\prime} \simeq 0$, nous verrons plus loin que les polarisations principales sont les polarisations circulaires droite et gauche; cette propriété n'est cependant pas générale et nous chercherons à déterminer dans les exemples qui vont suivre quelles sont dans chaque cas les polarisations principales correspondantes.

On vérifie (cf. $\$$ III.4.a) que $G$ est scalaire si l'on a simultanément $\bar{\sigma}_{\mathrm{f}}=\lambda 1\left(\bar{\sigma}_{\mathrm{f}}\right.$ scalaire $)$ et $H_{\mathrm{e}}^{\prime}=H_{\mathrm{f}}^{\prime}=0$. La modification de la polarisation lumineuse provient 
donc, d'une part du fait que $\bar{\sigma}_{\mathrm{f}}$ n'est en général pas scalaire, d'autre part de l'existence d'une structure énergétique dans l'état fondamental ou l'état excité. En pratique, pour jouer un rôle, cette structure ne doit pas être négligeable devant $\Delta+\Delta^{\prime}$ : nous avons vu en effet au $\S$ II 4 que dans (II.22) on peut négliger la contribution des valeurs de $\tau$ très supérieures à $1 / \Delta$ ou $1 / \Delta^{\prime}$; dans l'expression de $G(t), H_{\mathrm{e}, \mathrm{f}}^{\prime} \cdot \tau$ est donc au plus de l'ordre de $H_{\mathrm{e}, \mathrm{f}}^{\prime} /\left(\Delta+\Delta^{\prime}\right)$. Lorsque la condition

$$
H_{\mathrm{e}}^{\prime}, H_{\mathrm{f}}^{\prime} \ll \Delta+\Delta^{\prime}
$$

est réalisée, on peut remplacer les exponentielles $\mathrm{e}^{ \pm i H_{\mathrm{e}}^{\prime} \mathrm{f}} \cdot \tau$ par 1 , ce qui fait disparaître $H_{\mathrm{e}}^{\prime}$ et $H_{\mathrm{f}}^{\prime}$ de l'expression $\pi_{T}-\pi_{I}$.

Nous allons donc distinguer deux cas extrêmes :

1) $\bar{\sigma}_{\mathrm{f}} \neq \lambda 1$, mais $H_{\mathrm{e}}^{\prime}, H_{\mathrm{f}}^{\prime} \ll \Delta+\Delta^{\prime}$.

La modification de polarisation ne provient que de l'existence des différences de populations ou des « cohérences hertziennes » [2] dans l'état fondamental. L'effet correspondant sera appelé effet paramagnétique.

2) $\bar{\sigma}_{\mathrm{f}}=\lambda 1, H_{\mathrm{e}}^{\prime}$ ou $H_{\mathrm{f}}^{\prime}$ ne sont pas négligeables devant $\Delta+\Delta^{\prime}$. Ces conditions sont toujours réalisées lorsque l'état fondamental est diamagnétique (un seul sous-niveau $|\mu\rangle)$ et que la séparation énergétique dans l'état excité est suffisamment grande. On dit alors qu'il y a effet diamagnétique. Par extension, et même si l'état fondamental n'est pas diamagnétique, nous parlerons encore d'effet diamagnétique pour désigner l'influence de $H_{\mathrm{e}}^{\prime}$ et $H_{\mathrm{f}}^{\prime}$ sur $\pi_{T}-\pi_{\mathrm{I}}$.

Dans le paragraphe III, nous étudions le cas des isotopes impairs du mercure lorsque n'intervient que l'effet paramagnétique. Au paragraphe IV, nous étudions celui des isotopes pairs du mercure, où n'intervient que l'effet diamagnétique; nous calculons aussi dans ce cas l'effet sur la lumière transmise d'un champ de radiofréquence agissant sur l'état excité. Enfin, dans le paragraphe $\mathrm{V}$, nous abordons le cas des alcalins lorsque les deux effets paramagnétique et diamagnétique interviennent simultanément.

\section{EFFET PARAMAGNETIQUE}

Cas des isotopes impairs du mercure. - Dans le cas des isotopes impairs du mercure, la condition (III.0) est souvent largement réalisée, si l'on ne s'intéresse qu'à une composante hyperfine de la raie de résonance et si le champ magnétique n'est pas trop fort. Dans JP 1 et JP 2 [3], on suppose que c'est le cas. Nous pouvons donc, pour étudier l'effet paramagnétique, prendre un modèle identique à celui qui est utilisé dans ces articles.

1. Notations; Modèle de JP 1 ET JP 2. - L'état fondamental est un niveau de moment angulaire $I$. En présence d'un champ magnétique porté par l'axe Oz d'un trièdre direct Oxyz, on $\mathrm{a}: H_{\mathrm{f}}^{\prime}=\hbar \omega_{\mathrm{f}} I_{z}$ $\left(\omega_{\mathrm{f}}\right.$, pulsation de Larmor dans l'état fondamental $)$; les niveaux $\mid \mu>$ sont les sous-niveaux Zeeman d'énergies $\mu \hbar \omega_{\mathrm{f}}$. En pratique, nous avons toujours

$$
\omega_{\mathrm{f}} l / c \ll 1
$$

de sorte que nous pouvons remplacer dans (II.22) $\bar{\sigma}_{\mathrm{f}}(t)$ par $\sigma_{\mathrm{f}}(t)$ (cf. $\S \mathrm{II} .5$ ).

Nous ne considérons dans l'état excité qu'un niveau hyperfin de moment angulaire $F ; H_{\mathrm{e}}^{\prime}=\hbar \omega_{\mathrm{e}} F_{z} \quad\left(\omega_{\mathrm{e}}\right.$, pulsation de Larmor dans l'état excité). Nous supposons en effet que la fréquence centrale $\omega_{\mathrm{c}} / 2 \pi$ de la raie excitatrice n'est voisine que de la fréquence associée à la transition $F \leftrightarrow I$, et que la largeur $\Delta$ de cette raie est petite devant la structure hyperfine du niveau excité; il est alors possible de négliger les effets des niveaux hyperfins autres que $F$.

On suppose enfin que $\omega_{\mathrm{e}, \mathrm{f}} \ll \Delta+\Delta^{\prime}$.

2. Galcul de $\pi_{T}-\pi_{I^{*}}-$ Avec nos hypothèses, on peut remplacer dans (II.22) $H_{\mathrm{e}}^{\prime}$ et $H_{\mathrm{f}}^{\prime}$ par 0 . L'intégrale en $\tau$ se calcule alors aisément, et l'on obtient :

$$
G(t)=\alpha\left(\Gamma^{\prime} / 2+i \Delta E^{\prime}\right) B(t)
$$

avec les notations suivantes :

$$
\alpha=\frac{\omega_{c} l}{c} \frac{\mathscr{N} \hbar}{2 \varepsilon_{0} I}
$$

(où $I$ est l'intensité lumineuse incidente) est une constante homogène à un temps.

$\Gamma^{\prime}$ et $\Delta E^{\prime}$ sont les quantités déjà introduites dans JP 1 et JP 2; elles représentent respectivement l'élargissement et le déplacement de l'état fondamental dus à l'excitation optique, et sont données par :

$$
\begin{gathered}
\frac{\Gamma^{\prime}}{2}\left(\omega_{0}\right)+i \Delta E^{\prime}\left(\omega_{0}\right)=\frac{i q^{2}}{\hbar^{2}} \frac{|\langle F\|\mathbf{S}\| I\rangle|^{2}}{2 F+1} \\
\int I(\omega) \mathrm{d} \omega \int \frac{f(\mathbf{v}) \mathrm{d}^{3} \mathbf{v}}{\omega-\omega_{0}-\mathbf{k} \cdot \mathbf{v}+i \Gamma / 2}
\end{gathered}
$$

$q\langle F\|\mathbf{S}\| I\rangle$ est l'élément de matrice réduit du dipôle électrique $q \mathbf{S}$ entre les niveaux $F$ et $I$.

La matrice $B(t)$ est définie par :

$$
\left\langle\mathbf{e}_{\mathrm{i}}|B(t)| \mathbf{e}_{\mathrm{j}}\right\rangle=\operatorname{Tr}_{\mathrm{f}}\left\{\sigma_{\mathrm{f}}(t)\left(\mathbf{e}_{\mathrm{i}}^{*} \cdot \mathbf{D}\right) P_{\mathrm{e}}\left(\mathbf{e}_{\mathrm{j}} \cdot \mathbf{D}\right)\right\}
$$

D est un opérateur vectoriel hermitique sans dimensions, proportionnel à la partie angulaire de $q \mathbf{S}$; dans notre cas particulier, on peut définir $\mathbf{D}$ par les éléments de matrice de ses composantes standards $D_{q}$ dans les axes Oxyz en posant :

$$
\left\{\begin{array}{l}
\left\langle m\left|D_{q}\right| \mu\right\rangle=\langle I 1 \mu q \mid F m\rangle \\
\left\langle\mu\left|D_{q}\right| m\right\rangle=(-1)^{q}\left\langle m\left|D_{-q}\right| \mu\right\rangle .
\end{array}\right.
$$

On a : $B=B^{+}$, de sorte que (II.18) s'écrit : $\pi_{T}(t)-\pi_{I}=-\alpha \Gamma^{\prime} / 2\left[B\left(t-Z_{0} / c\right), \pi_{I}\right]_{+}$ $-i \alpha \Delta E^{\prime}\left[B\left(t-Z_{0} / c\right), \pi_{I}\right]$.

Nous voyons donc qu'avec les hypothèses de JP 1 et JP 2 l'équation générale (II.18) se simplifie considérablement. 
3. Discussion DE L'ÉQuATion (III.5). - a) Structure de l'équation. - Juxtaposons (III.5) avec l'équation (III.A.4) de la référence [2] :

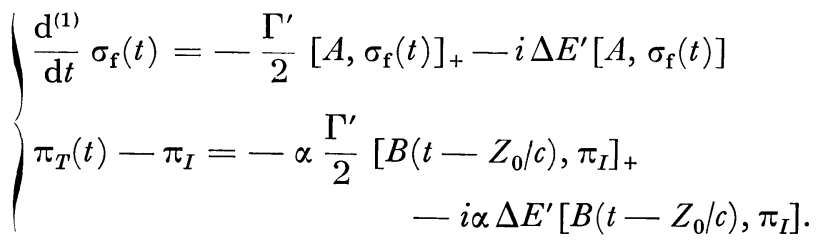

L'analogie entre les deux équations est frappante. Nous trouvons dans chacune d'elles : tout d'abord un anticommutateur multiplié par $\Gamma^{\prime}$, puis un commutateur multiplié par $\Delta E^{\prime}$. Seuls les anticommutateurs ont une trace non nulle; ils décrivent comment le processus d'absorption fait varier le nombre total d'atomes dans l'état fondamental $\left[\operatorname{Tr}\left(\mathrm{d}^{(\mathbf{1})} / \mathrm{d} t \sigma_{\mathrm{f}}\right)\right]$ et le nombre total de photons $\left[\operatorname{Tr}\left(\pi_{T}-\pi_{I}\right)\right]$. Les deux anticommutateurs traduisent donc l'effet des transitions réelles de résonance optique sur l'atome (durée de vie d'origine optique de l'état fondamental) et sur le photon (absorption d'énergie lumineuse). Quant aux commutateurs, ils traduisent l'effet des transitions virtuelles de résonance optique sur l'atome (déplacement optique de l'état fondamental) et sur le photon (modification de la vitesse de propagation associée à la dispersion).

Les matrices $A$ et $B$ sont sans dimensions. $\Gamma^{\prime}$ et $\Delta E^{\prime}$ sont proportionnels à l'intensité lumineuse $I$, mais ne dépendent pas du nombre d'atomes par unité de volume $\mathscr{N}$; par contre, $\alpha \Gamma^{\prime}$ et $\alpha \Delta E^{\prime}$ ne dépendent plus de $I$, mais sont proportionnels à $\mathscr{N}$. La variation relative de $\sigma_{f}$ dépend donc du nombre de photons, alors que celle de $\pi$ dépend du nombre d'atomes.

b) Étude des matrices $A$ et $B$.

$$
\left\{\begin{array}{l}
\left\langle\mu|A| \mu^{\prime}\right\rangle=\sum_{m}\left\langle\mu\left|\mathbf{e}_{\lambda_{0}}^{*} \cdot \mathbf{D}\right| m\right\rangle\left\langle m\left|\mathbf{e}_{\lambda_{0}} \cdot \mathbf{D}\right| \mu^{\prime}\right\rangle, \\
\left\langle\mathbf{e}_{\mathbf{i}}|B(t)| \mathbf{e}_{\mathbf{j}}\right\rangle \\
=\sum_{m \mu \mu^{\prime}} \sigma_{\mu^{\prime} \mu}(t)\left\langle\mu\left|\mathbf{e}_{\mathbf{i}}^{*} \cdot \mathbf{D}\right| m\right\rangle\left\langle m\left|\mathbf{\theta}_{\mathbf{j}} . \mathbf{D}\right| \mu^{\prime}\right\rangle . \quad \text { (III. }
\end{array}\right.
$$

La matrice $A$ agit dans l'espace des états de l'atome; c'est une matrice hermitique; Cohen-Tannoudji [2] a désigné par $|\alpha\rangle$ les états propres de $A$ (valeurs propres $p_{\alpha}$ ) et leur a donné l'interprétation physique suivante : lorsque l'atome est dans l'un des états $|\alpha\rangle$, il acquiert, du fait du processus d'absorption, une durée de vie $T_{p} / p_{\alpha}$ et subit un déplacement énergétique $\hbar p_{\alpha} \Delta E^{\prime}$ bien définis. La matrice $B(t)$ agit dans l'espace $\mathscr{2}$ des états de polarisation de la lumière; c'est une matrice hermitique qui possède deux vecteurs propres que nous désignerons par $|\beta\rangle$ (valeurs propres $\left.q_{\beta}\right)$ : ce sont les polarisations principales. Les états de polarisation $|\beta\rangle$ sont pour le photon ce que sont les états $|\alpha\rangle$ pour l'atome : lorsque le faisceau lumineux est dans l'un de ces deux états de polarisation, il subit une atténuation et un déphasage bien définis.

* Les matrices $A$ et $B$ sont définies positives ( $p_{\alpha}$, $\left.q_{\beta} \geqslant 0\right)$; quelle que soit la matrice densité $\sigma_{\mathrm{f}}(t)$, on peut dire qu'à tout instant le processus d'absorption porte des atomes de l'état fondamental vers l'état excité $\left[\operatorname{Tr}\left\{\mathrm{d}^{(1)} / \mathrm{d} t \sigma_{\mathrm{f}}(t)\right\}=-\Gamma^{\prime} \operatorname{Tr}\left\{A \sigma_{\mathrm{f}}(t)\right\} \leqslant 0\right]$, et que l'énergie du faisceau lumineux diminue $\left[\operatorname{Tr}\left(\pi_{T}-\pi_{I}\right)=-\Gamma^{\prime} \operatorname{Tr}\left\{B(t) \pi_{I}\right\} \leqslant 0\right]$.

Écrivons l'élément de matrice de $A$ en introduisant la matrice polarisation $\pi\left(\mathbf{e}_{\lambda_{0}}\right)=\left|\mathbf{e}_{\lambda_{0}}\right\rangle\left\langle\mathbf{e}_{\lambda_{0}}\right|$; $\left[\operatorname{Tr} \pi\left(\mathbf{e}_{\lambda_{0}}\right)=1, \pi_{I}=I \pi\left(\mathbf{e}_{\lambda_{0}}\right)\right] ;$ on pose :

$$
\pi\left(\mathbf{e}_{\lambda_{0}}\right)_{\mathbf{i}, \mathbf{j}}=\left\langle\mathbf{e}_{\mathbf{i}}\left|\pi\left(\mathbf{e}_{\lambda_{0}}\right)\right| \mathbf{e}_{\mathbf{j}}\right\rangle
$$

$A_{\mu \mu^{\prime}}=\left\langle\mu|A| \mu^{\prime}\right\rangle$ s'écrit alors :

$A_{\mu \mu^{\prime}}=\sum_{\mathbf{i}, \mathbf{j}, m}\left[\pi\left(\mathbf{e}_{\lambda_{0}}\right)\right]_{\mathbf{j}_{, \mathbf{i}}}\left\langle\mu\left|\mathbf{e}_{\mathbf{i}}^{*} \cdot \mathbf{D}\right| m\right\rangle\left\langle m\left|\mathbf{e}_{\mathbf{j}} \cdot \mathbf{D}\right| \mu^{\prime}\right\rangle$.

Comparons (III.6) et (III.7); la symétrie entre $A$ et $B$ est évidente; $A$ et $B$ peuvent s'obtenir en prenant la trace par rapport aux variables des photons ou des atomes d'une matrice $\mathscr{A}$ plus générale, correspondant à un opérateur agissant sur les deux types de variables, et définie par :

$\left\langle\mu \mathbf{e}_{\mathbf{i}}|\mathscr{A}| \mu^{\prime} \mathbf{e}_{\mathbf{j}}\right\rangle=\sum_{m}\left\langle\mu\left|\mathbf{e}_{\mathbf{i}}^{*} \cdot \mathbf{D}\right| m\right\rangle\left\langle m\left|\mathbf{e}_{\mathbf{j}} \cdot \mathbf{D}\right| \mu^{\prime}\right\rangle$.

$(\mathscr{A}$ n'est d'ailleurs pas un nouvel opérateur; $\left(\Gamma^{\prime} / 2+i \Delta E^{\prime}\right) \mathscr{A}$ est, à un coefficient réel près, la valeur que prend l'opérateur $K$ défini au $\S$ II.4 dans le cas de l'effet paramagnétique.)

On a :

$$
\left\{\begin{array}{l}
A=\operatorname{Tr}_{P}\left\{\pi_{I}\left(\mathbf{e}_{\lambda_{0}}\right) \mathscr{A}\right\} \\
B=\operatorname{Tr}_{\mathrm{f}}\left\{\sigma_{\mathrm{f}}(t) \mathscr{A}\right\}
\end{array}\right.
$$

$\operatorname{Tr}_{P}$ désigne l'opération de trace partielle effectuée sur les variables de polarisation de la lumière.

c) Calcul de $L_{A}\left(\mathbf{e}_{\lambda_{0}}\right)$. - Montrons enfin qu'il est possible de retrouver l'expression de

$$
L_{A}\left(\mathbf{e}_{\lambda_{0}}\right)=\Gamma^{\prime} \sum_{\mu \mu^{\prime}} A_{\mu \mu^{\prime}}\left(\sigma_{\mathrm{f}}\right)_{\mu^{\prime} \mu}
$$

quantité totale de lumière absorbée par la vapeur par unité de temps, qui est calculée dans JP 1 et JP 2; en l'absence d'analyseur, le signal optique est

$$
\begin{aligned}
\Delta I & =\operatorname{Tr}\left(\pi_{I}-\pi_{T}\right)=\operatorname{Tr}\left\{\left(\alpha \Gamma^{\prime} / 2\right)\left[B, \pi_{I}\right]_{+}\right\} \\
& =\alpha \Gamma^{\prime} I \operatorname{Tr}\left\{B\left|\mathbf{e}_{\lambda_{0}}\right\rangle\left\langle\mathbf{e}_{\lambda_{0}}\right|\right\}=\alpha \Gamma^{\prime} I\left\langle\mathbf{e}_{\lambda_{0}}|B| \mathbf{e}_{\lambda_{0}}\right\rangle
\end{aligned}
$$

en utilisant (III.6), il vient :

$\Delta I=\alpha \Gamma^{\prime} I \sum_{\mu \mu^{\prime}} A_{\mu \mu^{\prime}}\left(\sigma_{\mathrm{f}}\right)_{\mu^{\prime} \mu}=\alpha I L_{A}\left(\mathbf{e}_{\lambda_{0}}\right)$.

4. INFLuence Des Diverses obSeRVABLes DE L'ÉtAT FONDAMENTAL SUR LES PROPRIÉTÉS OPTIQUES DE LA VAPEUR DANS LE GAS DE L'EFFET PARAMAGNÉTIQUE. Les signaux optiques dépendent de $\bar{\sigma}_{\mathrm{f}}$ par l'intermédiaire de $G$ et permettent donc de mesurer un certain nombre d'observables relatives au niveau fondamental des atomes. Nous allons étudier dans le cas général quelles sont ces observables et leur influence sur $\pi_{T}$. Il n'est pas indispensable de préciser la nature des niveaux $|\mu\rangle$ pourvu que $\Delta \gg H_{\mathrm{f}}^{\prime}, H_{\mathrm{e}}^{\prime}$; cependant, nous prendrons souvent comme exemple le cas du mercure. 
Dans (III.5), seule la matrice $B(t)$ dépend de $\sigma_{\mathrm{f}} ;$ ses éléments de matrice $\left\langle\mathbf{e}_{\mathrm{i}}|B(t)| \mathbf{e}_{\mathrm{j}}\right\rangle$ sont les valeurs moyennes d'opérateurs de la forme $\left(\mathbf{e}_{\mathrm{i}}^{*}\right.$. D) $P_{\mathrm{e}}\left(\mathbf{e}_{\mathrm{j}}\right.$. D) ; i et j pouvant prendre chacun deux valeurs, $\pi_{T}-\pi_{I}$ dépend donc des valeurs moyennes dans l'état fondamental de quatre opérateurs. Ces opérateurs ne se transforment pas de manière simple par rotation; pour mettre en évidence l'influence sur $B(t)$ des propriétés de symétrie de $\bar{\sigma}_{\mathrm{f}}$, il est intéressant d'introduire de nouveaux opérateurs $\left(^{5}\right)$. Pour cela, on remarque que D est vectoriel, alors que le projecteur sur l'état excité $P_{\mathrm{e}}$ est scalaire; il est donc possible d'exprimer tous les éléments $\left\langle\mathbf{e}_{\mathrm{i}}|B(t)| \mathbf{e}_{\mathrm{j}}\right\rangle$ en fonction des valeurs moyennes $\left\langle T_{q}^{(k)}\right\rangle=\operatorname{Tr}_{\mathrm{f}}\left\{\bar{\sigma}_{\mathrm{f}}(t) T_{q}^{(k)}\right\}$ d'un opérateur tensoriel $(k=2,-2 \leqslant q \leqslant+2)$, d'un opérateur vectoriel $(k=1,-1 \leqslant q \leqslant+1)$ et d'un scalaire $(k=q=0)$ [5]. La définition des opérateurs $T_{q}^{(k)}$ en fonction des composantes standards de $\mathbf{D}$ relatives aux axes $O X Y Z$ est :

$T_{q}^{(k)}=\sum_{q^{\prime}+q^{\prime \prime}=q}\left\langle 11 q^{\prime} q^{\prime \prime} \mid k q\right\rangle D_{q^{\prime}} P_{e} D_{q^{\prime \prime}}$.

On a :

$$
(-1)^{q} T_{q}^{(k)}=\left[T_{-q}^{(k)}\right]^{+} .
$$

Soient $\mathrm{e}^{-i \theta} \cos \varphi, \mathrm{e}^{i \theta} \sin \varphi$ les composantes de $\mathbf{e}_{\mathbf{1}}$ sur les axes $\mathrm{OX}$ et $\mathrm{OY} ;-\mathrm{e}^{-i \theta} \sin \varphi$ et $\mathrm{e}^{i \theta} \cos \varphi$ celles de $\mathbf{e}_{2}$. On calcule :

$$
\begin{aligned}
\left(\mathbf{e}_{1}^{*} . \mathbf{D}\right) & P_{\mathrm{e}}\left(\mathbf{e}_{1} . \mathbf{D}\right)=\frac{1}{2}(\cos 2 \varphi-i \sin 2 \varphi \cos 2 \theta) T_{+2}^{(2)} \\
+ & \frac{1}{2}(\cos 2 \varphi+i \sin 2 \varphi \cos 2 \theta) T_{-2}^{(2)} \\
& -\frac{1}{\sqrt{6}}\left(T_{0}^{(2)}+\sqrt{2} T_{0}^{(0)}\right)+\frac{1}{\sqrt{2}} \sin 2 \varphi \sin 2 \theta T_{0}^{(1)}
\end{aligned}
$$

$$
\begin{aligned}
\left(\mathbf{e}_{1}^{*} . \mathbf{D}\right) & P_{\mathrm{e}}\left(\mathbf{e}_{2} . \mathbf{D}\right) \\
= & -\frac{1}{2}(\sin 2 \varphi-\sin 2 \theta+i \cos 2 \varphi \cos 2 \theta) T_{2}^{(2)} \\
& \quad-\frac{1}{2}(\sin 2 \varphi+\sin 2 \theta-i \cos 2 \varphi \cos 2 \theta) T_{-2}^{(2)} \\
+ & \frac{1}{\sqrt{2}}(\cos 2 \varphi \sin 2 \theta-i \cos 2 \theta) T_{0}^{(1)} . \quad \text { (III.14) }
\end{aligned}
$$

Les autres éléments de matrice de $B(t)$ se déduisent aisément de ceux-ci en changeant $\varphi$ en $(\varphi+\pi / 2)$.

La matrice $B(t)$ permet de calculer tous les signaux optiques des expériences que nous avons envisagées; $B(t)$ dépend des valeurs moyennes de quatre opérateurs $T_{0}^{(1)}, T_{-2}^{(2)}, T_{+2}^{(2)}$ et $\left[T_{0}^{(2)}+\sqrt{2} T_{0}^{(0)}\right]$, que la relation (III.12) permet d'exprimer en fonction de quatre observables. Ces observables sont liées aux axes OXYZ qui définissent la direction de propagation de la lumière, et nous connaissons leurs propriétés de

(5) Des techniques analogues sont utilisées dans la référence [4] pour l'étude de l'influence des observables de l'état excité sur la lumière de fluorescence. transformation par rotation. Dans le cas du mercure, le sens physique de ces observables est le suivant : $\left\langle T_{0}^{(0)}\right\rangle$ est proportionnel à la population globale de l'état fondamental $\left(\left\langle T_{0}^{(0)}\right\rangle\right.$ est constant $) ;\left\langle T_{0}^{(1)}\right\rangle$ est proportionnel à l'orientation de cet état dans la direction de propagation du faisceau lumineux (c'est-à-dire à $\left.\left\langle I_{Z}\right\rangle\right) ;\left\langle T^{(2)}\right\rangle$ à l'alignement (par exemple $\left\langle T_{0}^{(2)}\right\rangle$ est proportionnel à $\left.3\left\langle I_{Z}^{2}\right\rangle-I(I+1)\right)$.

La connaissance des valeurs moyennes de ces quatre observables permet de déterminer très rapidement les propriétés optiques de la vapeur : pour connaître par exemple les polarisations principales, il suffit, en remarquant que $B(t)$ est hermitique et que ces polarisations sont donc toujours orthogonales, de rechercher les valeurs de $\theta$ et $\varphi$ qui annulent l'expression de l'élément non diagonal $\left\langle\mathbf{e}_{\mathrm{i}}|B(t)| \mathbf{e}_{\mathrm{j}}\right\rangle$ obtenue à partir de (III.14).

Nous allons appliquer cette étude à un certain nombre de cas particuliers :

a) $\bar{\sigma}_{\mathrm{f}} \propto 1 . \bar{\sigma}_{\mathrm{f}}$ est invariant par rotation. Si $R$ est un opérateur de rotation dans l'espace des états, on a donc $: \bar{\sigma}_{\mathrm{f}}=R \bar{\sigma}_{\mathrm{f}} R^{+}$, et :

$$
\begin{aligned}
\left\langle T_{q}^{(k)}\right\rangle & =\operatorname{Tr}_{\mathrm{f}}\left\{R \bar{\sigma}_{\mathrm{f}} R^{+} T_{q}^{(k)}\right\}=\operatorname{Tr}_{\mathrm{f}}\left\{\bar{\sigma}_{\mathrm{f}} R^{+} T_{q}^{(k)} R\right\} \\
& =\left\langle R^{+} T_{q}^{(k)} R\right\rangle .
\end{aligned}
$$

$\left\langle T_{q}^{(k)}\right\rangle$ est donc invariant lorsque l'on fait subir une rotation à l'opérateur $T_{q}^{(k)}$; or, nous savons que par définition les opérateurs $T_{q}^{(k)}$ se transforment par rotation comme les harmoniques sphériques $Y_{q}^{(k)}$. Comme l'unique harmonique sphérique qui ne varie pas par rotation est $Y_{0}^{(0)}$, on a : $\left\langle T_{q}^{(k)}\right\rangle=0$ si $k$ et $q$ ne sont pas simultanément nuls.

On voit alors sur (III.13) et (III.14) que la matrice $B(t)$ est multiple de la matrice unité; l'absorption et la dispersion sont indépendantes de $\pi_{I}$; aucune polarisation n'est modifiée par traversée de la vapeur.

b) $\pi_{I} \propto 1$. On éclaire la vapeur en lumière non polarisée. On a : $\pi_{T}=\frac{I}{2}\left(1-\Gamma^{\prime}\right) B(t) . B(t)$ dépend de $\left\langle T^{(2)}\right\rangle$ et de $\left\langle T^{(1)}\right\rangle$, et la lumière transmise peut être partiellement polarisée si ces grandeurs ne sont pas nulles; l'analyse de cette polarisation permet donc de mesurer certaines des composantes de ces opérateurs.

Par contre, on voit sur (III.13) que la mesure de la lumière totale absorbée, proportionnelle à $\operatorname{Tr}[B(t)]$, ne permet d'atteindre que la combinaison

$$
\left\langle T_{0}^{(2)}+\sqrt{2} T_{0}^{(0)}\right\rangle ;
$$

sans analyseur ni polariseur, on peut détecter par exemple un alignement des atomes dans la direction OZ.

c) $\bar{\sigma}_{\mathrm{f}}$ est invariant par rotation autour d'un axe $\mathrm{Oz}$.

Effet Faraday paramagnétique : si la lumière se propage dans la direction $\mathrm{Oz}, \mathrm{OZ}$ et $\mathrm{Oz}$ sont confondus. Les seules harmoniques sphériques invariantes par rotation autour de $\mathrm{OZ}$ sont les $Y_{0}^{(k)}$, et on montre, par un 
raisonnement semblable à celui qui est fait en $a$ ), que $\left\langle T_{q}^{(k)}\right\rangle=0$ si $q \neq 0$. On utilise alors (III.14) pour trouver les polarisations principales; l'élément non diagonal de $B$ s'annule pour $\varphi=\pi / 4$ et $\theta=\pi / 4$, ce qui correspond aux polarisations circulaires droite et gauche. (III.13) nous montre que $\pi_{T}$ ne dépend plus que de deux observables : $\left\langle T_{0}^{(2)}+\sqrt{2} T_{0}^{(0)}\right\rangle$ qui apporte la même contribution aux deux indices principaux (absorption indépendante de $\pi_{I}$ ) $;\left\langle T_{0}^{(1)}\right\rangle$ qui est proportionnel à la différence entre les deux indices principaux : la vapeur possède donc un pouvoir rotatoire sur les polarisations planes proportionnel à $\Delta E^{\prime}\left\langle T_{0}^{(1)}\right\rangle$ et un dichroïsme circulaire proportionnel à $\Gamma^{\prime}\left\langle T_{0}^{(1)}\right\rangle$.

Biréfringence magnétique : si la lumière se propage perpendiculairement à $\mathrm{Oz}$, on peut supposer que les axes $\mathrm{OX}$ et $\mathrm{Oz}$ sont confondus. Les valeurs moyennes $\left\langle T_{q}^{(k)}\right\rangle$ sont donc invariantes par rotation autour de OX; or, si l'on considère une rotation $R_{X}(\pi)$ d'un angle $\pi$ autour de OX, on a [6] :

$$
R_{X}(\pi) T_{q}^{(k)} R_{X}(\pi)^{+}=\mathrm{e}^{-i k \pi} T_{-q}^{(k)} .
$$

On a donc :

$$
\left\langle T_{2}^{(2)}\right\rangle=\left\langle T_{-2}^{(2)}\right\rangle \quad \text { et } \quad\left\langle T_{0}^{(1)}\right\rangle=0 .
$$

On voit alors sur (III.14) que les polarisations principales sont les polarisations planes parallèles à $\mathrm{OX}$ et $\mathrm{OY}$, et que la différence entre leurs indices est proportionnelle à $\left\langle T_{2}^{(2)}\right\rangle$.

d) Supposons que $\left\langle T^{(2)}\right\rangle=0$; l'alignement dans l'état fondamental est nul, mais il peut subsister une orientation des atomes. Les deux seules observables dont dépend $B(t)$ sont $\left\langle T_{0}^{(1)}\right\rangle$ et $\left\langle T_{0}^{(0)}\right\rangle$; les deux polarisations principales sont les polarisations circulaires droite et gauche. La différence entre les deux indices correspondants est proportionnelle à $\left\langle T_{0}^{(1)}\right\rangle$ qui est ainsi responsable du pouvoir rotatoire et du dichroïsme circulaire de la vapeur.

Cas d'un spin $1 / 2\left({ }^{199} \mathrm{Hg}\right)$ : on a alors toujours $\left\langle T^{(2)}\right\rangle=0$; la seule observable dont dépend $B(t)$ est l'orientation de la vapeur dans la direction $\mathrm{OZ}$ de propagation de la lumière. Les expériences réalisées par Cohen-Tannoudji et Manuel [7] peuvent s'interpréter dans ce contexte; elles correspondent au cas où l'analyseur et le polariseur sont rectilignes. Prenons comme axe OX la direction du champ électrique de l'onde incidente, et supposons que l'analyseur laisse passer la composante du champ électrique qui fait un angle $\psi$ avec $\mathrm{OX}$; sa matrice $M_{\mathrm{a}}$ est donnée par (I.4). Calculons alors le signal de détection optique; nous trouvons :

$$
\begin{array}{r}
\Delta I=\operatorname{Tr}\left\{M_{\mathrm{a}}\left(\pi_{I}-\pi_{T}\right) M_{\mathrm{a}}^{+}\right\}=\operatorname{Tr}\left\{M_{\mathrm{a}}\left(\pi_{I}-\pi_{T}\right)\right\} \\
=-\frac{\alpha I}{\sqrt{3}} \Gamma^{\prime} \cos ^{2} \psi\left\langle T_{0}^{(0)}\right\rangle \\
-\alpha I \sqrt{2} \Delta E^{\prime} \sin \psi \cos \psi\left\langle T_{0}^{(1)}\right\rangle . \quad(\text { III } .17)
\end{array}
$$

Nous constatons que le seul terme qui puisse varier au cours d'une résonance magnétique est maximum si $\psi=\pi / 4$, et qu'il est proportionnel à $\Delta E^{\prime}$ : seules interviennent les transitions virtuelles. Cette dernière propriété est liée au fait que l'analyseur et le polariseur sont rectilignes et que l'épaisseur optique de la cellule est faible; lorsque ce n'est pas le cas, le signal de détection optique fait aussi intervenir un terme en $\Gamma^{\prime}$ (dichroïsme circulaire dû aux transitions réelles).

\section{EFFET DIAMAGNETIQUE}

Cas des isotopes pairs du mercure. - L'état fondamental $|\mu\rangle=|0\rangle$ est unique. Les niveaux excités $|m\rangle$ sont les trois sous-niveaux Zeeman $(m=-1,0$, +1 ) d'un niveau de moment cinétique donné $J=1$. Le champ magnétique statique est porté par l'axe $\mathrm{Oz}$ d'un trièdre direct Oxyz; il lui correspond l'hamiltonien $H_{\mathrm{e}}^{\prime}=\hbar \omega_{\mathrm{e}} J_{z} ;\left(J_{z}|m\rangle=m|m\rangle\right)$.

1. NOUS SUPPOSONS D'ABORD QU'IL N'Y A PAS DE GHAMP DE RADIOFRÉQUENCE. - La vapeur est donc placée dans les conditions où se produit l'effet Zeeman normal; la fréquence et la polarisation des raies lumineuses qu'elle émet lorsqu'elle est excitée dans ces conditions sont bien connues; notre étude consiste à calculer les conséquences de cet effet Zeeman normal sur l'indice de la vapeur.

a) Calcul de $\pi_{T}-\pi_{I}$. - On a :

$$
U_{0}^{\mathrm{e}}(t, t-\tau)=\mathrm{e}^{-i \omega_{\mathrm{e}} J_{z} \tau}
$$

Reportons (IV.1) dans (II.22); nous obtenons :

$G=\alpha \sum_{m=-1}^{+1}\left\{\frac{\Gamma^{\prime}}{2}\left(\omega_{0}+m \omega_{\mathrm{e}}\right)+i \Delta E^{\prime}\left(\omega_{0}+m \omega_{\mathrm{e}}\right)\right\} B_{m}$

$\operatorname{avec}\left({ }^{6}\right)$

$$
\left\langle\mathbf{e}_{\mathbf{i}}\left|B_{m}\right| \mathbf{e}_{\mathbf{j}}\right\rangle=\left\langle 0\left|\mathbf{e}_{\mathbf{i}}^{*} \cdot \mathbf{D}\right| m\right\rangle\left\langle m\left|\mathbf{e}_{\mathbf{j}} \cdot \mathbf{D}\right| 0\right\rangle .
$$

L'égalité $B_{m}=B_{m}^{+}$permet d'écrire :

$$
\begin{array}{r}
\pi_{T}-\pi_{I}=-\alpha \sum_{m}\left\{\frac{\Gamma^{\prime}}{2}\left(\omega_{0}+m \omega_{\mathrm{e}}\right)\left[B_{m}, \pi_{I}\right]_{+}\right. \\
\left.+i \Delta E^{\prime}\left(\omega_{0}+m \omega_{\mathrm{e}}\right)\left[B_{m}, \pi_{I}\right]\right\}
\end{array}
$$

La formule (IV.4) montre comment les diverses transitions atomiques $|0\rangle \leftrightarrow|m\rangle$ contribuent à la modification de la polarisation lumineuse : chacune de ces transitions fait intervenir les valeurs de $\Gamma^{\prime}$ et $\Delta E^{\prime}$ calculées pour la valeur de l'énergie qui leur correspond. Les polarisations principales sont en général quelconques; cependant, si la lumière se propage

( $\left.{ }^{6}\right) \Gamma^{\prime}\left(\omega_{0}\right), \Delta E^{\prime}\left(\omega_{0}\right)$ et $\mathbf{D}$ sont définis en remplaçant dans (III.2) et (III.4) $I$ et $\mu$ par zéro, $F$ par $J=1$ et $\langle F\|\mathbf{S}\| I\rangle$ par $\langle J\|\mathbf{S}\| 0\rangle$. 
parallèlement au champ magnétique, on voit sur (IV.3) que ce sont les polarisations circulaires $\sigma^{+}$ et $\sigma^{-}$, alors que, si la lumière se propage perpendiculairement au champ magnétique, ce sont les polarisations planes $\sigma$ et $\pi$.

On montre que $\Sigma\left\langle\mathbf{e}_{\mathrm{i}}\left|B_{m}\right| \mathbf{e}_{\mathbf{j}}\right\rangle=\left\langle\mathbf{e}_{\mathrm{i}} \mid \mathbf{e}_{\mathrm{j}}\right\rangle$; on a donc : $\sum_{m} B_{m}=1$; ; et si l'on fait dans (IV.4) $\omega_{\mathrm{e}}=0$, on obtient $\pi_{T}=\pi_{I}\left[1-\alpha \Gamma^{\prime}\left(\omega_{0}\right)\right]$. Ce résultat était prévisible : nous avons vu que, lorsqu'il n'y a ni effet paramagnétique ni effet diamagnétique, $\pi_{T}$ et $\pi_{I}$ correspondent à la même polarisation. La modification de polarisation lumineuse est donc due aux variations avec $\omega_{\mathrm{e}}$ de $\Gamma^{\prime}\left(\omega_{0} \pm \omega_{\mathrm{e}}\right)$ et $\Delta E^{\prime}\left(\omega_{0} \pm \omega_{\mathrm{e}}\right)$. L'étude précise de ces variations nécessite la connaissance exacte de la fonction $I(\omega)$ (forme de la raie excitatrice); soit $\omega_{\mathrm{e}}$ la pulsation centrale de la raie $I(\omega)$; nous savons qu'en pratique les variations avec $\omega_{\mathrm{e}}$ de $\Gamma^{\prime}\left(\omega_{0} \pm \omega_{\mathrm{e}}\right)$ et $\Delta E^{\prime}\left(\omega_{0} \pm \omega_{\mathrm{e}}\right)$ sont à peu près celles d'une courbe d'absorption ou de dispersion centrées en $\omega_{\mathrm{e}}= \pm\left(\omega_{\mathrm{c}}-\omega_{0}\right)$ et de largeur de l'ordre de $\Delta+\Delta^{\prime}$ (on suppose que l'on a $\Gamma \ll \Delta$ ou $\Delta^{\prime}$ ). Nous retrouvons donc ici un résultat annoncé au $\S$ II.4 : dans le cas d'une épaisseur optique faible, il est nécessaire, pour obtenir une modification de la polarisation lumineuse, de mettre la vapeur dans un champ magnétique intense, de sorte que $\omega_{\mathrm{e}}$ ne soit pas complètement négligeable devant $\Delta+\Delta^{\prime}$.

b) Calcul au premier ordre. - En pratique, la condition $\left(\omega_{\mathrm{e}} / \Delta\right)<1$ est souvent réalisée et l'on peut limiter le calcul au premier ordre en $\omega_{\mathrm{e}} / \Delta$ en remplaçant $\mathrm{e}^{-i \omega_{\mathrm{e}} J_{z} \tau}$ par $1-i \omega_{\mathrm{e}} J_{z} \tau$. On trouve alors, en utilisant (II.22) :

$$
\begin{array}{r}
\pi_{T}=\left[1-\alpha \Gamma^{\prime}\left(\omega_{0}\right)\right] \pi_{I}-\alpha \omega_{\mathrm{e}}\left\{\frac{\partial}{\partial \omega_{0}} \Gamma^{\prime}\left(\omega_{0}\right)\right\}\left[B^{\prime}, \pi_{I}\right]_{+} \\
-i \alpha \omega_{\mathrm{e}}\left\{\frac{\partial}{\partial \omega_{0}} \Delta E^{\prime}\left(\omega_{0}\right)\right\}\left[B^{\prime}, \pi_{I}\right] \quad(\mathrm{IV} .5)
\end{array}
$$

avec

$$
\left\langle\mathbf{e}_{\mathrm{i}}\left|B^{\prime}\right| \mathbf{e}_{\mathrm{j}}\right\rangle=\langle 0|\left(\mathbf{e}_{\mathrm{i}}^{*} \text {. D) } J_{z} P_{\mathrm{e}}\left(\mathbf{e}_{\mathrm{j}} \cdot \mathbf{D}\right)|0\rangle .\right.
$$

On montre aisément que :

$$
\begin{aligned}
& \left\langle\mathbf{e}_{\mathrm{i}}\left|B^{\prime}\right| \mathbf{e}_{\mathrm{j}}\right\rangle \\
& =\cos (\mathrm{OZ}, \mathrm{Oz})\left\langle 0\left|\left(\mathbf{e}_{\mathrm{i}}^{*} \cdot \mathbf{D}\right) J_{Z} P_{\mathrm{e}}\left(\mathbf{e}_{\mathrm{j}} \cdot \mathbf{D}\right)\right| 0\right\rangle .
\end{aligned}
$$

Lorsque la lumière se propage perpendiculairement au champ magnétique, l'effet diamagnétique est nul au premier ordre.

2. EFFET D'UN GHAMP DE RADIOFRÉQUENGE TOURNANT. - a) Le champ de radiofréquence tourne avec une vitesse angulaire $\Omega$ dans le plan $\mathrm{xOy}$ :

$$
H_{\mathrm{e}}^{\prime \prime}=\hbar \omega_{1}\left(J_{x} \cos \Omega t+J_{y} \sin \Omega t\right) \text {. }
$$

On peut calculer rigoureusement l'opérateur d'évolution $U_{0}^{\mathrm{e}}$, par exemple au moyen d'un passage dans le référentiel tournant :

$U_{0}^{\mathrm{e}}(t, t-\tau)=\mathrm{e}^{-i \Omega J_{z} t} \mathrm{e}^{-i\left[\left(\omega_{\mathrm{e}}-\Omega\right) J_{z}+\omega_{1} J_{x}\right] \tau} \mathrm{e}^{-i \Omega J_{z} \tau} \mathrm{e}^{i \Omega J_{z} t}$.
Au paragraphe II.4, nous avons négligé la dépendance en $t$ de $K$ en remplaçant dans (II.11) $U_{0}^{\prime \prime \text { e, f }}$ par 1, c'est-à-dire en négligeant l'action de la radiofréquence; cette approximation est valable lorsque la condition $\omega_{1} \ll \Delta+\Delta^{\prime}$ est réalisée. Ici nous supposons $\omega_{1}$ quelconque et nous tenons compte rigoureusement de l'action de la radiofréquence en reportant (IV.9) dans l'expression exacte (II.11); les égalités (II.12) et (II.19) nous donnent alors $G(t)$. Il est utile d'introduire les états propres $|p\rangle$ de la projection du moment cinétique $\boldsymbol{J}$ sur le « champ efficace 》:

$\left[\left(\omega_{\mathrm{e}}-\Omega\right) J_{z}+\omega_{1} J_{x}\right]|p\rangle=p \tilde{\omega}_{\mathrm{e}}|p\rangle$

avec $\quad \tilde{\omega}_{\mathrm{e}}=\sqrt{\left(\omega_{\mathrm{e}}-\Omega\right)^{2}+\omega_{1}^{2}}$

$$
\left\{\begin{aligned}
\mid p & = \pm 1\rangle=\frac{ \pm 1}{2 \tilde{\omega}_{\mathrm{e}}}\left\{\left(\omega_{\mathrm{e}}-\Omega \pm \tilde{\omega}_{\mathrm{e}}\right)|m=1\rangle\right. \\
& \left.+\sqrt{2} \omega_{1}|m=0\rangle-\left(\omega_{\mathrm{e}}-\Omega \mp \tilde{\omega}_{\mathrm{e}}\right)|m=-1\rangle\right\} \\
\mid p & =0\rangle=\frac{1}{\sqrt{2} \tilde{\omega}_{\theta}}\left\{-\omega_{1}|m=1\rangle\right. \\
& \left.+\sqrt{2}\left(\omega_{\mathrm{e}}-\Omega\right)|m=0\rangle+\omega_{1}|m=-1\rangle\right\} .
\end{aligned}\right.
$$

$G$ s'écrit :

$$
\begin{aligned}
& G(t)=\alpha \sum_{m m^{\prime} p}\left\{\left[\frac{\Gamma^{\prime}}{2}\left(\omega_{0}+m \Omega+p \tilde{\omega}_{\mathrm{e}}\right)\right.\right. \\
& \left.\quad+i \Delta E^{\prime}\left(\omega_{0}+m \Omega+p \tilde{\omega}_{\mathrm{e}}\right)\right] \\
& \left.\quad\left\langle m^{\prime} \mid p\right\rangle\langle p \mid m\rangle F_{m m^{\prime}}(l) \mathrm{e}^{i\left(m-m^{\prime}\right) \Omega t} B_{m m^{\prime}}\right\}
\end{aligned}
$$

avec

$$
\left\langle\mathbf{e}_{\mathbf{i}}\left|B_{m m^{\prime}}\right| \mathbf{e}_{\mathbf{j}}\right\rangle=\left\langle m\left|\mathbf{e}_{\mathbf{j}} \cdot \mathbf{D}\right| 0\right\rangle\left\langle 0\left|\mathbf{e}_{\mathbf{i}}^{*} \cdot \mathbf{D}\right| m^{\prime}\right\rangle
$$

et

$F_{m m^{\prime}}=\frac{\sin \left[\Omega\left(m-m^{\prime}\right) l / 2 c\right]}{\Omega\left(m-m^{\prime}\right) l / 2 c} \quad$ si $\quad m \neq m^{\prime} ; F_{m m}=1$.

Les expressions ainsi calculées nous permettent de déterminer exactement le signal optique dans tous les cas.

Les facteurs $F_{m m^{\prime}}(l)$ rendent compte de sa variation avec la longueur $l$ de la cellule; nous retrouvons dans le cas des alcalins des termes identiques et nous étudierons leur sens physique (cf. $\S$ V.4); nous ne le faisons donc pas ici, en supposant par exemple que l'on a $(\Omega l / c) \ll 1$, ce qui permet de remplacer tous les facteurs $F_{m m^{\prime}}(l)$ par 1 .

Les exponentielles $\mathrm{e}^{i\left(m-m^{\prime}\right) \Omega t}$ contiennent la dépendance en $t$ de $G(t)$; on voit qu'il peut apparaître dans le signal de détection optique des modulations aux pulsations $\Omega$ et $2 \Omega$; la dépendance en $t$ de $G(t)$ est particulièrement simple lorsque le faisceau lumineux se propage parallèlement au champ magnétique : on remarque en effet que

$$
\left\langle m\left|\mathrm{e}^{i \Omega J_{z} t}\left(\mathbf{e}_{\mathbf{j}} \cdot \mathbf{D}\right)\right| 0\right\rangle=\left\langle m\left|\tilde{\mathbf{e}}_{\mathbf{j}}(t) \cdot \mathbf{D}\right| 0\right\rangle
$$


où $\tilde{\mathbf{e}}_{\mathbf{j}}(t)$ est le vecteur obtenu en faisant tourner $\mathbf{e}_{\mathbf{j}} \mathrm{d}$ 'un angle - $\Omega t$ autour de $\mathrm{Oz}$; dans une base de deux polarisations tournant avec le champ de radiofréquence à la vitesse angulaire $\Omega, G$ est constant.

Les facteurs

$$
\Gamma^{\prime}\left(\omega_{0}+m \Omega+p \tilde{\omega}_{\mathrm{e}}\right) \text { et } \Delta E^{\prime}\left(\omega_{0}+m \Omega+p \tilde{\omega}_{\theta}\right)
$$

rendent compte des variations du signal de détection optique en fonction de la forme de la raie excitatrice $I(\omega)$; nous verrons que ce sont eux qui permettent aussi de déterminer la forme des courbes de résonance magnétique. Le choix des deux paramètres $\Gamma^{\prime}$ et $\Delta E^{\prime}$ qui est fait dans JP 1 et JP 2 pour décrire l'excitation optique est donc très utile puisqu'il permet de décrire simplement un grand nombre d'expériences différentes.

Si l'on a : $\tilde{\omega}_{\mathrm{e}} \ll \Delta+\Delta^{\prime}$, on peut négliger dans $\Gamma^{\prime}\left(\omega_{0}+m \Omega+p \tilde{\omega}_{\mathrm{e}}\right)$ et $\Delta E^{\prime}\left(\omega_{0}+m \Omega+p \tilde{\omega}_{\mathrm{e}}\right) \quad$ l'in- $^{\prime}$ fluence des quantités $p \tilde{\omega}_{\mathrm{e}}$; la sommation sur $p$ dans (IV.13) est alors très simple et on retrouve pour $G$ l'expression (IV.2) : la radiofréquence n'a aucune influence sur les signaux optiques. Si par contre $\tilde{\omega}_{\mathrm{e}}$ n'est pas totalement négligeable devant $\Delta+\Delta^{\prime}$, on voit sur (IV.13) qu'il faut considérer non seulement les états propres $|m\rangle$ de l'hamiltonien en l'absence de radiofréquence, mais aussi les états $|p\rangle$ et leurs énergies Zeeman $\hbar \tilde{\omega}_{\mathrm{e}}$ relatives au champ « efficace » qui interviennent dans $\Gamma^{\prime}$ et $\Delta E^{\prime}$.

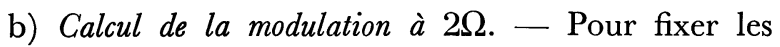
idées, nous allons calculer le terme de $G(t)$ qui oscille à la pulsation $2 \Omega$; il s'écrit :

$$
\begin{gathered}
G^{2 \Omega}=\alpha\left(\omega_{1} / 2 \tilde{\omega}_{\mathrm{e}}\right)^{2} B_{+1,-1} \Phi\left(\omega_{0}+\Omega, \tilde{\omega}_{\mathrm{e}}\right) \mathrm{e}^{2 i \Omega t} \\
+\alpha\left(\omega_{1} / 2 \tilde{\omega}_{\mathrm{e}}\right)^{2} B_{-1,+1} \Phi\left(\omega_{0}-\Omega, \tilde{\omega}_{\mathrm{e}}\right) \mathrm{e}^{-2 i \Omega t}
\end{gathered}
$$

$$
\begin{aligned}
\Phi\left(\omega_{0}, \tilde{\omega}_{\mathrm{e}}\right)=\frac{\Gamma^{\prime}}{2}\left(\omega_{0}\right. & \left.+\tilde{\omega}_{\mathrm{e}}\right)+i \Delta E^{\prime}\left(\omega_{0}+\tilde{\omega}_{\mathrm{e}}\right) \\
& +\frac{\Gamma^{\prime}}{2}\left(\omega_{0}-\tilde{\omega}_{\mathrm{e}}\right)+i \Delta E^{\prime}\left(\omega_{0}-\tilde{\omega}_{\mathrm{e}}\right) \\
& -\Gamma^{\prime}\left(\omega_{0}\right)-2 i \Delta E^{\prime}\left(\omega_{0}\right) .
\end{aligned}
$$

Intensité du champ de radiofréquence. - Nous avons vu plus haut que, lorsque l'on a : $\tilde{\omega}_{e} \ll \Delta+\Delta^{\prime}$, $G^{2 \Omega}$ est négligeable; nous ne considérerons donc que le cas où $\tilde{\omega}_{\mathrm{e}}$ n'est pas très petit devant $\Delta+\Delta^{\prime}$. Or $G^{2 \Omega}$ contient en facteur le terme $\left(\omega_{1} / \tilde{\omega}_{\mathrm{e}}\right)^{2}$; il faut donc aussi que $\omega_{1}$ ne soit pas négligeable devant $\Delta+\Delta^{\prime}$ : l'intensité du champ de radiofréquence nécessaire pour observer sur la lumière transmise une résonance magnétique dans l'état excité est donc considérablement augmentée par l'effet Doppler optique (rappelons que, dans le cas de la détection habituelle de cette résonance au moyen de la lumière de fluorescence, il suffit d'avoir $\left.\left(\omega_{1} / \Gamma\right) \simeq 1\right)$.

Forme des courbes de résonance magnétique. Nous pouvons obtenir la forme des courbes de résonance magnétique en faisant varier le champ magnétique statique appliqué sur l'atome : $\Omega$ est constant, $\omega_{e}$ varie. Le calcul exact est donc possible à partir de (IV.13) si l'on connaît les fonctions $\Gamma^{\prime}\left(\omega_{0}\right)$ et $\Delta E^{\prime}\left(\omega_{0}\right)$; nous nous contenterons ici de remarquer que ces courbes sont centrées à la résonance $\omega_{\mathrm{e}}=\Omega$ ( $G$ ne change pas lorsque l'on remplace $\omega_{\mathrm{e}}$ par $2 \Omega-\omega_{\mathrm{e}}$ ) et d'évaluer la largeur de ces courbes en supposant que la résonance n'est pas saturée (c'est-àdire que l'on a $\left.\omega_{1}<\Delta+\Delta^{\prime}\right)$. $\omega_{e}$ intervient par l'intermédiaire d'expressions du type

$$
\begin{aligned}
\left(1 / \tilde{\omega}_{\theta}\right)^{2}\left\{\Gamma^{\prime}\left(\omega_{0}+\Omega+\tilde{\omega}_{\theta}\right)+\Gamma^{\prime}\left(\omega_{0}+\Omega-\tilde{\omega}_{e}\right)\right. & \\
& \left.-2 \Gamma^{\prime}\left(\omega_{0}+\Omega\right)\right\}
\end{aligned}
$$

et si $\tilde{\omega}_{\mathrm{e}} \ll \Delta+\Delta^{\prime}$, cette expression est constante et vaut $\left(\mathrm{d}^{2} / \mathrm{d} \omega_{0}^{2}\right) \Gamma^{\prime}\left(\omega_{0}\right)$. Les variations de $G^{2 \Omega}$ ne sont donc notables que lorsque $\tilde{\omega}_{e}$ et donc $\omega_{e}$ varient de quantités qui sont de l'ordre de $\Delta+\Delta^{\prime}$; on voit que l'effet Doppler optique modifie profondément et élargit les courbes de résonance magnétique dans l'état excité (rappelons que, dans le cas de la détection de cette résonance au moyen de la lumière de fluorescence, la largeur de ces courbes est de l'ordre de $\Gamma$ ).

L'expérience que nous venons d'analyser est identique à une expérience de « double résonance » [8], à ceci près qu'au lieu d'utiliser la méthode classique qui consiste à mesurer la polarisation de la lumière de fluorescence, on mesure la polarisation de la lumière transmise par la cellule. Corney, Kibble et Series [9] ont étudié ce problème en supposant que la lumière se propage parallèlement au champ magnétique statique et que la cellule est placée entre deux polariseurs croisés. Nous avons généralisé leurs résultats en supposant que la direction de propagation de la lumière et l'analyseur A sont quelconques. Notons toutefois que le calcul de Series et al. suppose que l'épaisseur optique de la cellule n'est pas faible, alors que nous faisons ici l'hypothèse contraire (dans l'approximation des épaisseurs optiques faibles, on ne garde, comme nous l'avons vu au $\S$ I.6.e, que le terme du premier ordre en $\mathbf{E}^{R}$ qui correspond à l'interférence entre l'onde incidente et l'onde rayonnée par la cellule $\mathrm{G}$; en négligeant les termes d'ordres supérieurs, l'effet de la cellule C sur l'intensité transmise par l'analyseur est alors nul si le polariseur et l'analyseur sont croisés). Rappelons que la généralisation de nos résultats au cas des épaisseurs optiques quelconques est effectuée en Appendice.

Remarquons enfin qu'il est possible de calculer $\pi_{T}-\pi_{I}$ lorsque le champ de radiofréquence, au lieu d'être tournant, est linéaire. Dans ce cas, on ne peut plus calculer rigoureusement l'opérateur d'évolution $U$, mais on peut utiliser un développement de $U$ en série de puissances de $\omega_{1}$, et reporter ce développement dans (II .11). La lumière transmise peut alors comporter des modulations dont les pulsations sont des multiples de $\Omega$ supérieurs à $2 \Omega$.

\section{CAS DES ALCALNNS}

1. Notations. - L'état fondamental ${ }^{2} S_{1 / 2}$ des alcalins comporte deux niveaux hyperfins dont la différence d'énergie est $\hbar \Delta W$; nous les désignerons par $F\left(F=F_{1}\right.$ 
ou $F_{2}$ ). L'état excité comprend plusieurs niveaux hyperfins $\mathscr{F} ; \hbar \Delta \mathscr{W}$ est la différence d'énergie entre les niveaux $\mathscr{F}$ les plus éloignés. Si nous nous intéressons à la raie $D_{1}$ (transition ${ }^{2} S_{1 / 2} \leftrightarrow{ }^{2} P_{1 / 2}$ ), $\mathscr{F}$ peut prendre deux valeurs $\mathscr{F}_{1}$ et $\mathscr{F}_{2}$; par contre, si nous nous intéressons à la raie $D_{2}$ (transition ${ }^{2} S_{1 / 2} \leftrightarrow{ }^{2} P_{3 / 2}$ ), $\mathscr{F}$ peut prendre quatre valeurs $\mathscr{F}_{1}, \mathscr{F}_{2}, \mathscr{F}_{3}, \mathscr{F}_{4}$.

Soient $|F, \mu\rangle(-F \leqslant \mu \leqslant+F)$ les sous-niveaux Zeeman du niveau hyperfin $F ;|\mathscr{F}, m\rangle(-\mathscr{F} \leqslant m \leqslant$ $+\mathscr{F})$ ceux du niveau hyperfin $\mathscr{F} ; \omega_{F} / 2 \pi$ et $\omega_{\mathscr{F}} / 2 \pi$ sont respectivement les fréquences de Larmor à l'intérieur de ces niveaux (le champ magnétique est supposé suffisamment faible pour n'introduire aucun découplage hyperfin). Il est commode d'introduire les projecteurs : $P_{\mathrm{F}}=\sum_{-F \leqslant \mu \leqslant+F}|F, \mu\rangle\langle F, \mu|$ sur le niveau $F$ $P_{\mathscr{F}}=\sum_{\mathscr{F} \leqslant m \leqslant \mathscr{F}}|\mathscr{F}, m\rangle\langle\mathscr{F}, m|$ sur le niveau $\mathscr{F}$.

Nous supposerons que dans tous les cas la largeur $\Delta+\Delta^{\prime}$ est très grande devant les diverses précessions de Larmor $\omega_{F}$ et $\omega_{\mathscr{F}}$; par contre, $\Delta$ et $\Delta^{\prime}$ peuvent être de l'ordre de $\Delta W$ et de $\Delta \mathscr{W}$.

Pour le césium par exemple, à température ordinaire : $\Delta^{\prime} \simeq \frac{1}{30} \Delta W \simeq \frac{1}{3} \Delta \mathscr{W}\left(D_{1}\right)$, alors que pour le sodium : $\Delta^{\prime} \simeq \Delta W \simeq 10 \Delta \mathscr{W}\left(D_{1}\right)$.

Nous sommes donc conduits à tenir compte de l'effet diamagnétique relatif à l'état fondamental, alors que jusqu'ici nous l'avions négligé. Si $\bar{\sigma}_{\mathrm{f}}$ n'est pas scalaire, nous sommes dans un cas où interviennent simultanément l'effet paramagnétique et l'effet diamagnétique.

Nous allons calculer dans ce qui suit $\pi_{T}-\pi_{I}$. Rappelons que la généralisation des calculs de JP 1 et JP 2 (évolution de la matrice densité atomique) au cas des alcalins a été faite par M. A Bouchiat [10].

2. Calcul de $\pi_{T}-\pi_{I}$. Soit $\hbar \omega_{F \mathscr{F}}^{0}$ la différence d'énergie entre les niveaux $|F, \mu=0\rangle$ et $|\mathscr{F}, m=0\rangle$; le calcul nous conduit à introduire les quantités $\Gamma_{F \mathscr{F}}^{\prime}=\Gamma^{\prime}\left(\omega_{0}=\omega_{F \mathscr{F}}^{0}\right)$ et $\Delta E_{F \mathscr{F}}^{\prime}=\Delta E^{\prime}\left(\omega_{0}=\omega_{F \mathscr{F}}^{0}\right)$.

$\Gamma^{\prime}\left(\omega_{0}\right)$ et $\Delta E^{\prime}\left(\omega_{0}\right)$ sont définis par :

$$
\begin{array}{r}
\frac{\Gamma^{\prime}}{2}\left(\omega_{0}\right)+i \Delta E^{\prime}\left(\omega_{0}\right)=\frac{i q^{2}}{\hbar^{2}} \frac{\left|\left\langle J_{\mathrm{e}}\|\mathbf{S}\| J_{\mathrm{f}}\right\rangle\right|^{2}}{2 J_{\mathrm{e}}+1} \\
\int I(\omega) \mathrm{d} \omega \int \frac{f(\mathbf{v}) \mathrm{d}^{3} \mathbf{v}}{\omega-\omega_{0}-\mathbf{k v}+i \Gamma / 2}
\end{array}
$$

où $q\left\langle J_{\mathrm{e}}\|\mathbf{S}\| J_{\mathrm{f}}\right\rangle$ est l'élément de matrice réduit du dipôle électrique $q \mathbf{S}$ entre les niveaux ${ }^{2} S_{1 / 2}$ et ${ }^{2} P_{1 / 2}$ (raie $D_{1}$ ) ou ${ }^{2} P_{3 / 2}$ (raie $\left.D_{2}\right)$. $\Gamma_{F \mathscr{F}}^{\prime}$ et $\Delta E_{F \mathscr{F}}^{\prime}$ sont les valeurs des paramètres $\Gamma^{\prime}$ et $\Delta E^{\prime}$ pour l'énergie de la transition $F \leftrightarrow \mathscr{F}$. Nous allons introduire, comme au $\S$ III.2, un opérateur $\mathbf{D}$ proportionnel à la partie angulaire du dipôle électrique $q \mathbf{S}$ et qui, comme $q \mathbf{S}$, n'agit que sur les variables de l'électron et pas sur celles du noyau; on vérifie qu'on peut définir ses composantes standards de la manière suivante :

$$
\begin{gathered}
\left\langle\mathscr{F}, m\left|D_{q}\right| F, \mu\right\rangle=\sum_{m_{I}, m_{J}, m_{J}^{\prime}}\left\langle\mathscr{F}, m \mid I J_{\mathrm{e}} m_{I} m_{J}\right\rangle \\
\left\langle J_{\mathrm{f}} 1 m_{J}^{\prime} q \mid J_{\mathrm{e}} m_{J}\right\rangle\left\langle I J_{\mathrm{f}} m_{I} m_{J}^{\prime} \mid F, \mu\right\rangle \\
\left\langle F, \mu\left|D_{q}\right| \mathscr{F}, m\right\rangle=(-1)^{q}\left\langle\mathscr{F}, m\left|D_{-q}\right| F, \mu\right\rangle
\end{gathered}
$$

$m_{I}$ et $m_{J}$ sont les valeurs propres de $I_{z}$ et $J_{z}$, composantes sur $\mathrm{Oz}$ du moment angulaire nucléaire et électronique.

Il est utile d'introduire les matrices $B_{F \mathscr{F}}(t)$ définies par :

$\left\langle\mathbf{e}_{\mathrm{i}}\left|B_{F \mathscr{F}}(t)\right| \mathbf{e}_{\mathrm{j}}\right\rangle=\operatorname{Tr}_{\mathrm{f}}\left\{P_{F} \bar{\sigma}_{\mathrm{f}}(t) P_{F}\left(\mathbf{e}_{\mathrm{i}}^{*} \cdot \mathbf{D}\right) P_{\mathscr{F}}\left(\mathbf{e}_{\mathrm{j}} \cdot \mathbf{D}\right)\right\}$.

$B_{F \mathscr{F}}(t)$ est la valeur de la matrice $B(t)$ lorsque l'on ne considère que les niveaux hyperfins $F$ et $\mathscr{F}$; c'est une matrice hermitique qui dépend des populations et des « cohérences Zeeman » à l'intérieur du niveau $F$.

Il est nécessaire, pour calculer $G(t)$, d'introduire d'autres matrices $C_{\mathscr{F}}(t)$ définies par :

$\left\langle\mathbf{e}_{\mathbf{i}}\left|C_{\mathscr{F}}(t)\right| \mathbf{e}_{\mathrm{j}}\right\rangle=\operatorname{Tr}_{\mathrm{f}}\left\{P_{F_{1}} \bar{\sigma}_{\mathrm{f}}(t) P_{F_{\mathbf{2}}}\left(\mathbf{e}_{\mathrm{i}}^{*} \cdot \mathbf{D}\right) P_{\mathscr{F}}\left(\mathbf{e}_{\mathrm{j}} \cdot \mathbf{D}\right)\right\}$.

$C_{\mathscr{F}}(t)$ n'est pas hermitique; on a :

$\left\langle\mathbf{e}_{\mathrm{i}}\left|C_{\mathscr{F}}^{+}(t)\right| \mathbf{e}_{\mathrm{j}}\right\rangle=\operatorname{Tr}_{\mathrm{f}}\left\{P_{F_{\mathbf{2}}} \bar{\sigma}_{\mathrm{f}}(t) P_{F_{1}}\left(\mathbf{e}_{\mathbf{i}}^{*} \cdot \mathbf{D}\right) P_{\mathscr{F}}\left(\mathbf{e}_{\mathrm{j}} \cdot \mathbf{D}\right)\right\}$

et on pose :

$$
\begin{aligned}
C_{\mathscr{F}}^{R}(t) & =\frac{1}{2}\left[C_{\mathscr{F}}(t)+C_{\mathscr{F}}^{+}(t)\right] \\
C_{\mathscr{F}}^{I}(t) & =\frac{1}{2 i}\left[C_{\mathscr{F}}(t)-C_{\mathscr{F}}^{+}(t)\right] .
\end{aligned}
$$

$C_{\mathscr{F}}(t)$ dépend seulement des « cohérences hyperfines 》 entre les niveaux $F_{1}$ et $F_{2}$, et s'annule lorsque ces cohérences n'existent pas.

On obtient alors :

$$
\begin{aligned}
\pi_{T}-\pi_{I}=-\alpha \sum_{\mathscr{F}}\left\{\frac{1}{2} \Gamma_{F_{1} \mathscr{F}}^{\prime}\left[B_{F_{1} \mathscr{F}}(t), \pi_{I}\right]_{+}+i \Delta E_{F_{1} \mathscr{F}}^{\prime}\left[B_{F_{1} \mathscr{F}}(t), \pi_{I}\right]\right. \\
+\frac{1}{2} \Gamma_{F_{2} \mathscr{F}}^{\prime}\left[B_{F_{2} \mathscr{F}}(t), \pi_{I}\right]_{+}+i \Delta E_{F_{2} \mathscr{F}}^{\prime}\left[B_{F_{2} \mathscr{F}}(t), \pi_{I}\right] \\
+\left[\frac{1}{2}\left(\Gamma_{F_{1} \mathscr{F}}^{\prime}+\Gamma_{F_{2} \mathscr{F}}^{\prime}\right) C_{\mathscr{F}}^{R}(t)+\left(\Delta E_{F_{2} \mathscr{F}}^{\prime}-\Delta E_{F_{1} \mathscr{F}}^{\prime}\right) C_{\mathscr{F}}^{I}(t), \pi_{I}\right]_{+} \\
\left.+i\left[\left(\Delta E_{F_{1} \mathscr{F}}^{\prime}+\Delta E_{F_{2} \mathscr{F}}^{\prime}\right) C_{\mathscr{F}}^{R}(t)+\frac{1}{2}\left(\Gamma_{F_{1} \mathscr{F}}^{\prime}-\Gamma_{F_{2} \mathscr{F}}^{\prime}\right) C_{\mathscr{F}}^{I}(t), \pi_{I}\right]\right\}
\end{aligned}
$$


qui est l'équation fondamentale de notre problème dans le cas des alcalins. Les quatre premiers termes du second membre de (V.11) proviennent de la simple juxtaposition des termes d'effet paramagnétique à l'intérieur de chacun des niveaux $F_{1}$ et $F_{2}$ considérés comme isolés. Par contre, les termes suivants sont nouveaux : ils apparaissent lorsqu'il y a des cohérences hyperfines et oscillent à toutes les fréquences correspondant aux transitions

$$
\left|F_{1}, \mu\right\rangle \leftrightarrow\left|F_{2}, \mu^{\prime}\right\rangle .
$$

3. Influence des diverses observables de l'état fondamental. - a) CAS GÉNÉRAL. - Dans (V.11), $\bar{\sigma}_{\mathrm{f}}(t)$ intervient par l'intermédiaire des matrices $B_{F \mathscr{F}}(t)$ et $C_{\mathscr{F}}(t)$; on voit sur (V.7) et (V.8) que ces matrices font intervenir des valeurs moyennes d'opérateurs du type :

$$
P_{F^{r}}\left(\mathbf{e}_{\mathbf{i}}^{*} . \mathbf{D}\right) P_{\mathscr{F}}\left(\mathbf{e}_{\mathrm{j}} . \mathbf{D}\right) P_{F^{\prime \prime}}
$$

$\mathrm{i}, \mathrm{j}=1,2 ; F, F^{\prime}=F_{1}, F_{2} ; \mathscr{F}=\mathscr{F}_{1}, \mathscr{F}_{2} \quad\left(\right.$ raie $D_{1}$ ) ou $=\mathscr{F}_{1}, \mathscr{F}_{2}, \mathscr{F}_{3}, \mathscr{F}_{4}$ (raie $D_{2}$ ).

Le signal de détection optique dépend donc des valeurs moyennes des opérateurs définis en (V.12). De même qu'au paragraphe III.4, il est utile de remarquer que $P_{I^{\prime}}$ et $P_{\mathscr{F}}$ sont invariants par rotation et d'introduire les opérateurs tensoriels :

$$
T_{q}^{(k)}(\mathscr{F})=\sum_{q^{\prime}+q^{\prime \prime}=q}\left\langle 11 q^{\prime} q^{\prime \prime} \mid k q\right\rangle D_{q^{\prime}} P_{\mathscr{F}} D_{q^{\prime \prime}}
$$

Les calculs du paragraphe III.4 se généralisent aisément; il suffit de remplacer dans (III.13) et (III.14) les opérateurs $T_{q}^{(k)}$ par $T_{q}^{(k)}(\mathscr{F})$ et $B$ par $B_{F \mathscr{F}}, C_{\mathscr{F}}^{R}$ ou $C_{\mathscr{F}}^{I}$. Les observables dont dépend $\pi_{T}-\pi_{I}$ peuvent donc s'exprimer en fonction des opérateurs $P_{F} T_{q}^{(k)}(\mathscr{F}) P_{F^{\prime}}$

Les deux opérateurs $T_{q}^{(k)}(\mathscr{F})$ et $P_{F} T_{q}^{(k)}(\mathscr{F}) P_{H^{\prime \prime}}$ ont la même nature tensorielle. D'après le théorème de Wigner-Eckart, deux opérateurs de la forme $P_{F} T_{q}^{(k)}(\mathscr{F}) P_{F^{\prime}}$ ne différant que par la valeur de $\mathscr{F}$ sont proportionnels; toutes les quantités $\left\langle P_{F} T_{q}^{(k)}(\mathscr{F}) P_{F^{\prime}}\right\rangle$ dont dépend $\pi_{T}-\pi_{I}$ ne sont donc pas indépendantes. L'étude qui a été faite au paragraphe III. 4 des relations qui existent entre les symétries de $\bar{\sigma}_{\mathrm{f}}$ lors de diverses opérations de rotation et les polarisations principales, s'applique sans changement au cas des alcalins. Par contre, le sens physique des observables qui interviennent peut être plus complexe que dans le cas du mercure; par exemple, $\left\langle P_{F} T_{0}^{(0)}(\mathscr{F}) P_{F}\right\rangle$ est proportionnel à la population totale du niveau hyperfin $F$ qui n'est pas constante.

b) CAs ou $\Delta+\Delta^{\prime} \gg \Delta \mathscr{W}$. - La structure hyperfine dans l'état excité n'est pas résolue; c'est par exemple le cas du sodium. (Comme $\Delta W$ est à peu près dix fois plus grand que $\Delta \mathscr{W}$, nous ne supposons pas forcément que $\Delta+\Delta^{\prime}$ est grand devant $\Delta W$.) $\Gamma_{F \mathscr{F}}^{\prime}$ et $\Delta E_{F \mathscr{F}}^{\prime}$ sont alors indépendants de $\mathscr{F}$; soient $\Gamma_{1,2}^{\prime}$ et $\Delta E_{1,2}^{\prime}$ leurs valeurs pour $F=F_{1}, F_{2}$. L'équation (V.11) s'écrit :

$$
\begin{aligned}
\pi_{T}-\pi_{I}= & -(\alpha / 2) \Gamma_{1}^{\prime}\left[B_{1}(t), \pi_{I}\right]_{+}-i \alpha \Delta E_{1}^{\prime}\left[B_{1}(t), \pi_{I}\right] \\
& -(\alpha / 2) \Gamma_{2}^{\prime}\left[B_{2}(t), \pi_{I}\right]_{+}-i \alpha \Delta E_{2}^{\prime}\left[B_{2}(t), \pi_{I}\right] \\
& -(\alpha / 2)\left(\Gamma_{1}^{\prime}+\Gamma_{2}^{\prime}\right)\left[C^{R}(t), \pi_{I}\right]_{+} \\
& -\alpha\left(\Delta E_{2}^{\prime}-\Delta E_{1}^{\prime}\right)\left[C^{I}(t), \pi_{I}\right]_{+} \\
& -i \alpha\left(\Delta E_{1}^{\prime}+\Delta E_{2}^{\prime}\right)\left[C^{R}(t), \pi_{I}\right] \\
& -i(\alpha / 2)\left(\Gamma_{1}^{\prime}-\Gamma_{2}^{\prime}\right)\left[C^{I}(t), \pi_{I}\right] \quad(\mathrm{V} .14)
\end{aligned}
$$

avec

$$
\left\langle\mathbf{e}_{\mathrm{i}}\left|B_{1}(t)\right| \mathbf{e}_{\mathrm{j}}\right\rangle=\operatorname{Tr}_{\mathrm{f}}\left\{P_{F_{1}} \bar{\sigma}_{\mathrm{f}}(t) P_{F_{1}}\left(\mathbf{e}_{\mathrm{i}}^{*} \cdot \mathbf{D}\right) P_{\mathrm{e}}\left(\mathbf{e}_{\mathrm{j}} \cdot \mathbf{D}\right)\right\}
$$

$$
\left\langle\mathbf{e}_{\mathrm{i}}\left|B_{2}(t)\right| \mathbf{e}_{\mathrm{j}}\right\rangle=\operatorname{Tr}_{\mathrm{f}}\left\{P_{F_{\mathbf{z}}} \bar{\sigma}_{\mathrm{f}}(t) P_{F_{\mathbf{2}}}\left(\mathbf{e}_{\mathrm{i}}^{*} \text {. D }\right) P_{\mathrm{e}}\left(\mathbf{e}_{\mathrm{j}} \cdot \mathbf{D}\right)\right\}
$$

$\left\langle\mathbf{e}_{\mathrm{i}}|C(t)| \mathbf{e}_{\mathrm{j}}\right\rangle=\operatorname{Tr}_{\mathrm{f}}\left\{P_{F_{1}} \bar{\sigma}_{\mathrm{f}}(t) P_{F_{\mathbf{2}}}\left(\mathbf{e}_{\mathrm{i}}^{*} . \mathbf{D}\right) P_{\mathrm{e}}\left(\mathbf{e}_{\mathrm{j}} \cdot \mathbf{D}\right)\right\}$

$P_{\mathrm{e}}=\sum_{\mathscr{F}} P_{\mathscr{F}}$ étant le projecteur sur l'ensemble des niveaux excités, on a encore : $P_{\mathrm{e}}=\sum_{m_{I} m_{J}}\left|m_{I} m_{J}\right\rangle\left\langle m_{I} m_{J}\right|$.

$\pi_{T}-\pi_{I}$ s'exprime maintenant en fonction des valeurs moyennes dans l'état fondamental d'opérateurs du type $P_{F} T_{q}^{(k)} P_{F^{\prime}}, T_{q}^{(k)}$ étant défini par :

$$
T_{q}^{(k)}=\sum_{q^{\prime}+q^{\prime \prime}=q}\left\langle 11 q^{\prime} q^{\prime \prime} \mid k q\right\rangle D_{q^{\prime}} P_{\mathrm{e}} D_{q^{\prime \prime}}
$$

(V.18) diffère de (V.13) par le remplacement de $P_{\mathscr{F}}$ par $P_{\mathrm{e}}$.

On remarque que $P_{\mathrm{e}}=\sum_{m_{I} m_{J}}\left|m_{I} m_{J}\right\rangle\left\langle m_{I} m_{J}\right|$ est un opérateur scalaire vis-à-vis séparément des variables nucléaires et électroniques : il ne change pas lorsque l'on fait tourner l'électron seul (alors que $P_{\mathscr{F}}$ n'est invariant que lorsque l'on effectue la même rotation sur l'électron et le noyau). L'opérateur $T_{q}^{(k)}$ défini en (V.18) est donc comme D un opérateur scalaire vis-à-vis des variables nucléaires; sa nature tensorielle est la même si l'on considère que la rotation porte sur l'électron et le noyau, ou sur l'électron seul.

Or le niveau fondamental des alcalins est un niveau de moment cinétique électronique $J=1 / 2$; les éléments de matrice des opérateurs $T_{\alpha}^{(2)}$ sont donc tous nuls, et l'on a $\left\langle P_{F} T_{q}^{(2)} P_{F^{\prime}}\right\rangle=0$. En effectuant une décomposition du type de (III.13) et (III.14), on arrive au résultat suivant :

- Chacune des deux matrices $B_{1}(t)$ et $B_{2}(t)$ ne dépend plus que des valeurs moyennes de deux observables : $\left\langle P_{F} T_{0}^{(1)} P_{F^{*}}\right\rangle$ proportionnel à l'orientation électronique à l'intérieur du niveau hyperfin $F$ dans la direction $\mathrm{OZ} ;\left\langle P_{F} T_{0}^{(0)} P_{F}\right\rangle$ proportionnel à la population totale du niveau $F$.

- La matrice $C(t)$ dépend des parties réelle et imaginaire de $\left\langle P_{F_{1}} T_{0}^{(1)} P_{F_{2}}\right\rangle$; on a en effet

$$
P_{F_{1}} T_{0}^{(0)} P_{F_{2}}=P_{F_{1}} P_{F_{2}} T_{0}^{(0)}=0 \text {. }
$$

$\left\langle P_{F_{1}} T_{0}^{(1)} P_{F_{2}}\right\rangle$ représente la partie de l'aimantation électronique qui ne dépend que des cohérences hyperfines. 
En remarquant que la somme des populations des niveaux $F_{1}$ et $F_{2}$ est constante, on constate que $\pi_{T}-\pi_{I}$ ne dépend plus que de cinq observables hermitiques vectorielles ou scalaires de l'état fondamental. Ainsi, dans le cas d'un alcalin, la lumière transmise par la vapeur ne peut pas dépendre d'observables tensorielles d'ordre supérieur à 1 lorsque la structure hyperfine dans l'état excité n'est pas résolue. Nous généralisons ainsi un résultat de [11, 12].

Un raisonnement identique à celui du $\S$ III.4.d nous montre que les quatre matrices $B_{1}(t), B_{2}(t)$, $C^{R}(t)$ et $C^{I}(t)$ ont les mêmes polarisations propres : ce sont les polarisations circulaires droite et gauche qui sont toujours les polarisations principales. On aboutit donc au résultat suivant qui est simple, mais pas évident : lorsque la structure hyperfine dans l'état excité n'est pas résolue, une vapeur alcaline ne peut présenter qu'un pouvoir rotatoire sur les polarisations planes et un dichroïsme circulaire (et ceci même en présence de cohérences hyperfines, auquel cas la rotation du plan de polarisation et le dichrö̈sme sont modulés à la pulsation $\Delta W)$.

Remarquons enfin que $T^{(2)}$ étant nul, $\pi_{T}-\pi_{I}$ ne peut dépendre que des populations de $\sigma_{\mathrm{f}}$ et des " cohérences » entre des niveaux $|\mu\rangle$ et $\left|\mu^{\prime}\right\rangle$ avec $\mu-\mu^{\prime}=0, \pm 1$. On voit donc que, si l'on effectue une expérience de "faisceau croisé » [13] sur un alcalin, on n'obtient pas de modulations à $2 \omega_{F_{1}}$ ou $2 \omega_{F_{2}}$ lorsque la structure hyperfine dans l'état excité n'est pas résolue.

c) CAs ou $\Delta+\Delta^{\prime} \gg \Delta \mathscr{W}$ et $\Delta W$. - Les valeurs de $\Gamma^{\prime}$ et $\Delta E^{\prime}$ pour toutes les transitions hyperfines sont les mêmes : $\Gamma_{1}^{\prime}=\Gamma_{2}^{\prime}=\Gamma^{\prime}$ et $\Delta E_{1}^{\prime}=\Delta E_{2}^{\prime}=\Delta E^{\prime}$. Il n'y a donc plus d'effet diamagnétique, et nous sommes dans le cas qui a déjà été étudié au paragraphe III.4; nous allons voir cependant que dans le cas des alcalins certains résultats se simplifient.

On a :

$\pi_{T}-\pi_{I}=-\left(\alpha \Gamma^{\prime} / 2\right)\left[B(t), \pi_{I}\right]_{+}-i \alpha \Delta E^{\prime}\left[B(t), \pi_{I}\right]$ avec

$$
B(t)=B_{1}(t)+B_{\mathbf{2}}(t)+C(t)+C^{+}(t) .
$$

Les éléments de matrice de $B(t)$ sont donc :

$$
\begin{aligned}
\left\langle\mathbf{e}_{\mathrm{i}}|B(t)| \mathbf{e}_{\mathrm{j}}\right\rangle & =\operatorname{Tr}_{\mathrm{f}}\left\{\left[P_{F_{\mathbf{1}}}+P_{F_{\mathbf{2}}}\right] \bar{\sigma}_{\mathrm{f}}(t)\left[P_{F_{1}}+P_{F_{2}}\right]\right. \\
& \left.\left(\mathbf{e}_{\mathrm{i}}^{*} \cdot \mathbf{D}\right) P_{\mathrm{e}}\left(\mathbf{e}_{\mathrm{j}} \cdot \mathbf{D}\right)\right\} \\
& =\operatorname{Tr}_{\mathrm{f}}\left\{\bar{\sigma}_{\mathrm{f}}(t)\left(\mathbf{e}_{\mathrm{i}}^{*} \cdot \mathbf{D}\right) P_{\mathrm{e}}\left(\mathbf{e}_{\mathrm{j}} \cdot \mathbf{D}\right)\right\} .
\end{aligned}
$$

Ces éléments ne dépendent plus que de deux quantités qui sont les valeurs moyennes dans l'état fondamental (et non plus à l'intérieur du niveau $F_{1}$ ou $F_{2}$ ) de deux opérateurs n'agissant que sur les variables électroniques $(\mathrm{cf} . \S \mathrm{b}):\left\langle T_{0}^{(0)}\right\rangle$ qui est proportionnel à la population globale de l'état fondamental (on retrouve ainsi le fait que, lorsque $\Delta \gg \Delta W, \Delta \mathscr{W}$, on ne peut pas détecter optiquement une différence de populations hyperfines dans l'état fondamental $[10,11]) ;\left\langle T_{0}^{(1)}\right\rangle$ qui est proportionnel à l'orientation électronique dans la direction OZ. Les polarisations principales sont toujours les polarisations circulaires droite et gauche, la différence entre les indices principaux ne dépendant que de $\left\langle T_{0}^{(1)}\right\rangle$.

d) On montrerait de manière analogue, en utilisant le fait que le niveau fondamental des alcalins est un niveau de moment orbital $L=0$, que, lorsque la vapeur est éclairée avec la même intensité sur les raies $D_{1}$ et $D_{2}\left[\Gamma^{\prime}\left(D_{1}\right)=\Gamma^{\prime}\left(D_{2}\right) ; \Delta E^{\prime}\left(D_{1}\right)=\Delta E^{\prime}\left(D_{2}\right)\right]$, $\pi_{T}-\pi_{I}$ est indépendante de $\pi_{I}[10,11]$.

4. Influence de la longueur de la cellule. - Supposons que $\omega_{F_{1}}=\omega_{F_{2}}=0$; les cohérences hyperfines de $\sigma_{\mathrm{f}}$ oscillent à la pulsation $\Delta W$. Soit $\lambda_{H}=2 \pi c / \Delta W$ la longueur d'onde associée à la transition hyperfine. Cette longueur d'onde varie suivant l'alcalin considéré, et il peut arriver que $\lambda_{H}$ soit du même ordre de grandeur que la longueur $l$ de la cellule. Ainsi, pour le césium par exemple, $\lambda_{H} \simeq 3 \mathrm{~cm}$. Nous sommes alors dans le cas où il n'est pas possible de remplacer $\bar{\sigma}_{\mathrm{f}}(t)$ par $\sigma_{\mathrm{f}}(t)$ (cf. $\S$ II.5); il faut utiliser la formule exacte (II.21), c'est-à-dire tenir compte du temps de propagation de la lumière à l'intérieur de la cellule. Pour simplifier, nous négligerons la dépendance en $Z$ de $\sigma_{\mathrm{f}}(Z, t)$. Calculons à partir de (II.21) le terme de $\bar{\sigma}_{\mathrm{f}}(t)$ qui oscille à la pulsation $\Delta W$ :

$$
\begin{gathered}
P_{F_{1}} \bar{\sigma}_{\mathrm{f}}(t) P_{F_{2}}=\frac{1}{l} \int_{-l / 2}^{l / 2} P_{F_{1}} \sigma_{\mathrm{f}}\left(t_{0}\right) P_{F_{2}} \mathrm{e}^{i \Delta W\left(t-t_{0}+Z / c\right)} \mathrm{d} Z \\
\left.=\mathrm{e}^{i \Delta W\left(t-t_{0}\right)} \frac{\sin \left(\pi l / \lambda_{H}\right)}{\pi l / \lambda_{H}} P_{F_{1}} \sigma_{\mathrm{f}}\left(t_{0}\right) P_{F_{2}} . \quad \text { V } .20\right)
\end{gathered}
$$

D'où :

$$
\begin{aligned}
& \text { D'où : } \\
& \left\langle\mathbf{e}_{\mathrm{i}}|C(t)| \mathbf{e}_{\mathrm{j}}\right\rangle=\mathrm{e}^{i \Delta W\left(t-t_{0}\right)} \frac{\sin \left(\pi l / \lambda_{H}\right)}{\pi l / \lambda_{H}} \\
& \qquad \operatorname{Tr}_{\mathrm{f}}\left\{P_{F_{1}} \sigma_{\mathrm{f}}\left(t_{0}\right) P_{F_{\mathbf{2}}}\left(\mathbf{e}_{\mathrm{i}}^{*} . \mathbf{D}\right) P_{\mathrm{e}}\left(\mathbf{e}_{\mathrm{j}} . \mathbf{D}\right)\right\} .
\end{aligned}
$$

La partie modulée du signal optique est proportionnelle à $\alpha C(t)$; sa dépendance en $l$ est donc donnée par la fonction périodique $\sin \left(\pi l / \lambda_{H}\right)$. L'effet des phénomènes d'interférence entre les champs électriques rayonnés par les divers points de la cellule apparaît clairement; nous voyons par exemple que, lorsque l est un multiple entier de la longueur d'onde $\lambda_{H}$, la modulation à la pulsation $\Delta \mathrm{W}$ disparaît.

On peut interpréter ce résultat de la manière suivante : nous avons vu (cf. § II.3) que la polarisabilité du milieu est modulée par la précession hyperfine dans l'état fondamental; le dipôle électrique de la vapeur et le champ $\mathbf{E}^{R}$ rayonné en avant sont donc aussi modulés par cette précession. Lorsque $l=\lambda_{H}$, on peut décomposer la cellule en deux demi-cellules de longueur $\lambda_{H} / 2$, le temps de propagation de la lumière d'une demi-cellule à l'autre étant $\lambda_{H} / 2 c$, c'est-à-dire une demi-période hyperfine. Les modulations des champs rayonnés en avant par les deux demi-cellules sont alors en opposition de phase et se détruisent par interférence.

Le cas où $\sigma_{\mathrm{f}}(Z, t)$ dépend de $Z$ peut se traiter de manière analogue, les idées physiques restant les mêmes. 
5. Effets nouveaux dus à la présence simultanée d'effets paramagnétique et diamagnétique. - Nous supposons $\Delta \gg \Gamma, \Delta \mathscr{W}$, mais pas $\Delta \gg \Delta W$. Dans l'équation (V.14) ce sont les termes proportionnels à $C^{I}(t)$ qui introduisent des effets nouveaux par rapport à ceux que nous avons déjà étudiés plus haut à propos de l'effet paramagnétique pur ou de l'effet diamagnétique pur. Ces termes ne sont en effet non nuls que si l'on a à la fois effet paramagnétique (les " cohérences hyperfines » doivent être différentes de zéro, puisque $C^{I}(t)$ leur est proportionnel) et effet diamagnétique $\left(\Gamma_{1}^{\prime}-\Gamma_{2}^{\prime}, \Delta E_{1}^{\prime}-\Delta E_{2}^{\prime}\right.$ doivent être non nuls, ce qui interdit à $\Delta$ d'être très grand devant $\Delta W$ ).

Dans les termes en $C^{I}(t)$, les rôles que jouent habituellement $\Gamma^{\prime}$ et $\Delta E^{\prime}$ (cf. $\oint$ III.3.a) sont inversés : l'anticommutateur associé aux effets de l'absorption est multiplié par $\Delta E_{1}^{\prime}-\Delta E_{2}^{\prime}$; le commutateur associé aux effets de dispersion est multiplié par $\Gamma_{1}^{\prime}-\Gamma_{2}^{\prime}$.

Pour bien illustrer le caractère nouveau des termes en $C^{I}(t)$, prenons un exemple précis. Supposons que :

$$
\left\{\begin{array}{l}
\Gamma_{1}^{\prime} \simeq \Gamma_{2}^{\prime} \simeq 0 \\
\Delta E_{1}^{\prime}-\Delta E_{2}^{\prime} \neq 0 .
\end{array}\right.
$$

La condition $(V .22)$ est réalisée si la raie excitatrice (de pulsation centrale $\omega_{c}$ ) ne coïncide avec aucune des deux composantes hyperfines (de pulsation $\omega_{1}^{0}$ et $\left.\omega_{2}^{0} ; \omega_{1}^{0}-\omega_{2}^{0}=\Delta W\right)$. Ceci n'est possible que si :

$$
\left\{\begin{array}{l}
\Delta+\Delta^{\prime} \ll \Delta W \\
\left|\omega_{c}-\omega_{1}^{0}\right|,\left|\omega_{c}-\omega_{2}^{0}\right| \gg \Delta+\Delta^{\prime} .
\end{array}\right.
$$

$\Delta E_{1}^{\prime}$ et $\Delta E_{2}^{\prime}$ étant alors proportionnels à $1 /\left(\omega_{c}-\omega_{1}^{0}\right)$ et $1 /\left(\omega_{c}-\omega_{2}^{0}\right)$ ne diffèrent en valeur relative de façon importante que si :

$$
\omega_{c}-\omega_{1}^{0} \lesssim \omega_{2}^{0}-\omega_{2}^{0}=\Delta W .
$$

(Ainsi, si la raie excitatrice tombe au milieu des composantes hyperfines, $\omega_{1}^{0}>\omega_{c}>\omega_{2}^{0}, \Delta E_{1}^{\prime}$ et $\Delta E_{2}^{\prime}$ sont de signes opposés.)

Les conditions (V.24) et (V.26) expriment physiquement que, dans l'état fondamental, le mouvement propre de l'atome est rapide : la période hyperfine $2 \pi / \Delta W$ est courte devant le temps de passage $1 / \Delta$ des trains d'ondes du faisceau lumineux [2]; elle n'est pas grande devant les temps $1 /\left(\omega_{c}-\omega_{1}^{0}\right), 1 /\left(\omega_{c}-\omega_{2}^{0}\right)$ que durent les transitions virtuelles effectuées à partir de $F_{1}$ et $F_{2}$. Dans ces conditions, (V.14) s'écrit :

$$
\begin{gathered}
\pi_{T}-\pi_{I}=-i \alpha \Delta E_{1}^{\prime}\left[B_{1}(t), \pi_{I}\right]-i \alpha \Delta E_{2}^{\prime}\left[B_{2}(t), \pi_{I}\right] \\
-\alpha\left(\Delta E_{2}^{\prime}-\Delta E_{1}^{\prime}\right)\left[C^{I}(t), \pi_{I}\right]_{+} \\
-i \alpha\left(\Delta E_{1}^{\prime}+\Delta E_{2}^{\prime}\right)\left[C^{R}(t), \pi_{I}\right] . \quad(\mathrm{V} .27) \\
\operatorname{Tr}\left(\pi_{T}-\pi_{I}\right)=-2 \alpha\left(\Delta E_{2}^{\prime}-\Delta E_{1}^{\prime}\right) \operatorname{Tr}\left\{C^{I}(t) \pi_{I}\right\} .
\end{gathered}
$$

S'il n'y a pas de cohérences hyperfines, c'est-à-dire si l'atome est dans l'un des niveaux $F_{1}$ ou $F_{2}, C^{I}(t)=0$, $\operatorname{Tr}\left(\pi_{T}-\pi_{I}\right)=0$; il n'y a pas d'absorption. Ceci est normal : la conservation de l'énergie interdit à un atome dans $F_{1}$ ou $F_{2}$ d'absorber le photon incident (condition (V.22)). Par contre, si l'atome est dans une superposition linéaire de $F_{1}$ et $F_{2}, C^{I}(t)$ n'est pas nul et il apparaît une absorption d'énergie lumineuse (cf. (V .28)) proportionnelle à $\Delta E_{1}^{\prime}-\Delta E_{2}^{\prime}$, et modulée puisque $C^{I}(t)$ oscille à la pulsation $\Delta W$; de plus, $\operatorname{Tr}\left(\pi_{T}-\pi_{I}\right)$ oscille autour de la valeur zéro : à certains instants l'intensité lumineuse après la cellule peut être plus grande que s'il n'y avait pas de cellule. Par contre, la valeur moyenne de $\operatorname{Tr}\left(\pi_{T}-\pi_{I}\right)$ sus un temps long devant $1 / \Delta W$ est nulle. Les trainr d'ondes du faisceau lumineux ont une extension spatiale de l'ordre de $c / \Delta$. Comme $\frac{1}{\Delta} \gg \frac{1}{\Delta W}$, l'énergie totale contenue dans le train d'ondes n'a pas varié après traversée de la cellule. Le seul effet de la vapeur a été de redistribuer de façon périodique l'énergie à l'intérieur du train d'ondes, la distance entre deux maxima ou minima consécutifs étant $c / \Delta W$.

La situation analysée ici est donc nettement différente de celle qui a été étudiée plus haut (effet paramagnétique pur) où $\operatorname{Tr}\left(\pi_{T}-\pi_{I}\right)$ était constamment négatif ou nul (cf. $\S$ III.3.b), nul lorsque $\Gamma^{\prime}=0$. Ceci provenait de ce que, dans ce cas, l'atome n'avait pas le temps d'évoluer pendant le temps de passage du train d'ondes lumineuses $\left(\Delta \gg \omega_{\mathrm{f}}\right)$.

Notons que, lorsque (V.22) et (V.23) sont réalisées, on retrouve dans le calcul de l'évolution de la matrice densité atomique des termes analogues à ceux que nous avons obtenus pour $\pi_{T}-\pi_{I}[10]$.

Soulignons enfin que, même si l'on peut négliger $\Gamma_{2}^{\prime}$ et $\Delta E_{2}^{\prime}$ devant $\Gamma_{1}^{\prime}$ et $\Delta E_{1}^{\prime}$, il n'est pas correct lorsqu'il existe des cohérences hyperfines de calculer $\pi_{T}-\pi_{I}$ en considérant le niveau $F_{1}$ comme isolé : dans le terme $\Delta E_{1}^{\prime}\left[C^{I}(t), \pi_{I}\right]_{+}$de l'équation (V.14) interviennent en effet explicitement dans $C^{I}(t)$ les éléments de matrice du dipôle électrique entre le niveau $F_{2}$ et l'état excité.

Conclusion. - Nous pouvons résumer ainsi les résultats obtenus dans ces deux articles :

La variation de polarisation lumineuse après traversée de la vapeur, $\pi_{T}-\pi_{\mathrm{I}}$, a pu être calculée explicitement en fonction des divers paramètres du problème. En plus de la lumière totale absorbée $\Delta I=\operatorname{Tr}\left(\pi_{I}-\pi_{T}\right)$, nous pouvons ainsi calculer quantitativement n'importe quel signal de détection optique utilisant la lumière transmise par la vapeur, obtenu par exemple en observant cette dernière à travers un analyseur.

L'équation à laquelle nous aboutissons pour $\pi_{T}-\pi_{I}$ présente une structure très voisine de celle qui donne l'évolution de la matrice densité atomique $\sigma_{\mathrm{f}}$ sous l'influence du processus d'excitation optique. Nous avons analysé en grand détail cette analogie.

Nous avons également mis en évidence le lien existant entre les propriétés de symétrie de $\sigma_{\mathrm{f}}$ et les polarisations principales de la vapeur, et exprimé les signaux 
optiques en fonction d'un nombre relativement restreint d'observables de l'état fondamental.

Enfin, dans le cas des alcalins, nous prévoyons deux effets nouveaux :

La modulation à la fréquence hyperfine de la lumière transmise peut disparaître lorsque la longueur de la cellule est de l'ordre de la longueur d'onde associée à la transition hyperfine.

L'existence de cohérences hyperfines peut, dans certains cas, entraîner une modulation du flux lumineux transmis par la vapeur, même si la raie excitatrice ne coïncide avec aucune des composantes hyperfines de la raie de résonance optique.

\section{APPENDICE}

Cas des épaisseurs optiques non faibles. - Les calculs exposés dans ces deux articles ont été menés en supposant l'épaisseur optique de la vapeur faible; les signaux optiques sont alors reliés simplement aux observables de l'état fondamental (c'est d'ailleurs une des raisons pour laquelle la plupart des expériences de pompage optique sont réalisées dans ces conditions). Il est cependant possible de reprendre les calculs en s'affranchissant de cette condition. Le champ électrique total au point $Z$ à l'instant $t,\left|\mathscr{E}_{\omega} T(Z, t)\right\rangle$, est la somme du champ incident $\left|\mathscr{E}_{\omega} I\right\rangle$ et du champ rayonné par les atomes compris dans toutes les tranches $Z^{\prime}, Z^{\prime}+\mathrm{d} Z^{\prime}$, et excités non plus par le champ $\left|\mathscr{E}_{\omega} \boldsymbol{I}\right\rangle$ mais par le champ $\left|\mathscr{E}_{\omega}^{T}\left(Z^{\prime}, t-\frac{Z-Z^{\prime}}{c}\right)\right\rangle$. Nous avons donc, en utilisant (II.10) :

$$
\begin{aligned}
& \left|\mathscr{E}_{\omega}^{T}(Z, t)\right\rangle=\left|\mathscr{E}_{\omega} I\right\rangle-\frac{\mathscr{N} q^{2} \omega}{2 \varepsilon_{0} c \hbar} \int_{-\infty}^{Z} \mathrm{~d} Z^{\prime} \\
& \operatorname{Tr}_{\mathrm{f}}\left\{\sigma_{\mathrm{f}}\left[Z^{\prime}, t-\frac{Z-Z^{\prime}}{c}\right] K_{\omega}\left(t-\frac{Z-Z^{\prime}}{c}\right)\right\} \\
& \left|\mathscr{E}_{\omega}^{T}\left(Z^{\prime}, t-\frac{Z-Z^{\prime}}{c}\right)\right\rangle .
\end{aligned}
$$

La sommation est limitée entre $-\infty$ et $Z$, car les contributions à $\left|\mathscr{E}_{\omega}^{T}(Z, t)\right\rangle$ des atomes compris entre $Z$ et $+\infty$ s'annulent par interférences destructives. Nous pouvons résoudre (VI.1) par itération :

$$
\left|\mathscr{E}_{\omega}^{T}(Z, t)\right\rangle=M_{\omega}(Z, t)\left|\mathscr{E}_{\omega} I\right\rangle
$$

avec

$$
\begin{aligned}
& M_{\omega}(Z, t) \\
& =1+\sum_{n=1}^{\infty}\left(-\frac{\mathscr{N} q^{2} \omega}{2 \varepsilon_{0} c \hbar}\right)^{n} \int_{-\infty}^{Z} \mathrm{~d} Z_{1} \int_{-\infty}^{Z_{1}} \mathrm{~d} Z_{2} \ldots \int_{-\infty}^{Z_{n-1}} \mathrm{~d} Z_{n} \\
& \operatorname{Tr}_{\mathrm{f}}\left\{\sigma_{\mathrm{f}}\left[Z_{1}, t-\frac{Z-Z_{1}}{c}\right] K_{\omega}\left(t-\frac{Z-Z_{1}}{c}\right)\right\} \ldots \\
& \operatorname{Tr}_{\mathrm{f}}\left\{\sigma_{\mathrm{f}}\left[Z_{n}, t-\frac{Z-Z_{n}}{c}\right] K_{\omega}\left(t-\frac{Z-Z_{n}}{c}\right)\right\} .
\end{aligned}
$$

A l'ordre 1, on obtient (II.13) en posant $Z=Z_{0}$ et en utilisant le fait que $\sigma_{\mathrm{f}}\left(Z^{\prime}, t\right)$ est nul à l'extérieur de l'intervalle $-l / 2 \leqslant Z^{\prime} \leqslant+l / 2$. Remarquons l'analogie qui existe entre (VI.1) et la forme intégrale de l'équation de Schrödinger, et entre (VI.3) et le développement de l'opérateur d'évolution associé à cette équation en série de Neumann-Liouville de la perturbation [14]. On peut faire correspondre à chacun des termes du développement (VI.3) un diagramme où la « perturbation » (diffusion du champ électrique par les atomes de la tranche $Z^{\prime}, Z^{\prime}+\mathrm{d} Z^{\prime}$ ) agit $n$ fois; la variable $Z$ joue dans (VI.3) le rôle que joue la variable $t$ dans le développement de NeumannLiouville. On peut sommer formellement la série (VI.3) et obtenir pour $Z=Z_{0}$ (point d'observation à l'extérieur de la cellule) :

$M_{\omega}\left(Z_{0}, t\right)=P_{Z} \exp \left[-\frac{\mathscr{N} q^{2} \omega}{2 \varepsilon_{0} c \hbar} \int_{-l / 2}^{+l / 2} \mathrm{~d} Z\right.$

$\left.\operatorname{Tr}_{\mathrm{f}}\left\{\sigma_{\mathrm{f}}\left(Z, t-\frac{Z_{0}-Z}{c}\right) K_{\omega}\left(t-\frac{Z_{0}-Z}{c}\right)\right\}\right]$

où $P_{Z}$ est l'opérateur qui, dans chacun des termes du développement de l'exponentielle, range les opérateurs $\sigma_{\mathrm{f}}(Z, t) K_{\omega}(Z, t)$ par ordre de $Z$ décroissant de gauche à droite.

On obtient alors $\pi_{T}$ en effectuant une sommation $\operatorname{sur} \omega$ :

$\pi_{T}(t)=\int I(\omega) M_{\omega}\left(Z_{0}, t\right)\left|\mathbf{e}_{\lambda_{0}}\right\rangle\left\langle\mathbf{e}_{\lambda_{0}}\right| M_{\omega}^{+}\left(Z_{0}, t\right) \mathrm{d} \omega$.

Cette sommation se simplifie si la largeur de la raie excitatrice est faible $\left(\Delta \ll \Delta^{\prime}\right) ; M_{\omega}\left(Z_{0}, l\right)$ ne dépend alors pratiquement pas de $\omega$ sur l'intervalle où $I(\omega)$ n'est pas nul, et on a :

$$
\pi_{T}(t) \simeq M_{\omega_{c}}\left(Z_{0}, t\right) \pi_{I} M_{\omega_{c}}^{+}\left(Z_{0}, t\right) .
$$

L'expression (VI.4) est difficile à utiliser dans le cas général; elle se simplifie dans les deux cas suivants où l'opérateur $P_{Z}$ disparaît :

1) Effet diamagnétique $\left(\sigma_{\mathrm{f}} \propto 1\right)$, et absence de radiofréquence $\left(K_{\omega}\right.$ ne dépend alors pas de $\left.t\right)$. On a, dans ce cas :

$$
M_{\omega}=\exp \left[-\frac{\mathscr{N} q^{2} \omega l}{2 \varepsilon_{0} c \hbar} \operatorname{Tr}_{\mathrm{f}}\left\{K_{\omega}\right\}\right]=\exp \left[-G_{\omega}\right] .
$$

La matrice $G_{\omega}$ est donnée par l'équation (IV .2) où $\Gamma^{\prime}$ et $\Delta E^{\prime}$ sont calculés en supposant que la raie excitatrice est fine $\left(\Delta \ll \Delta^{\prime}\right)$ et centrée en $\omega_{c}=\omega$. L'équation (VI.7) a déjà été écrite par Omont [15] dans son étude de l'effet de la dispersion anormale sur la diffusion multiple.

2) On suppose que $\sigma_{\mathrm{f}}$ est indépendante de $Z$ et que le temps de propagation $l / c$ de la lumière à travers 
la cellule est très court devant les temps d'évolution de $\sigma_{\mathrm{f}}(t)$ et de $K(t)$. On peut alors dans (VI.4) négliger la dépendance en $Z$ (par l'intermédiaire de $t$ ) de $\sigma_{\mathrm{f}}$ et $K_{\omega}$. On a alors :

$$
M_{\omega}\left(Z_{0}, t\right)=\exp \left[-G_{\omega}\left(t-Z_{0} / c\right)\right]
$$

avec

$$
G_{\omega}(t)=\frac{\mathscr{N} q^{2}}{2 \varepsilon_{0} \hbar} \frac{\omega l}{c} \operatorname{Tr}_{\mathrm{f}}\left\{\sigma_{\mathrm{f}}(t) K_{\omega}(t)\right\} .
$$

Dans ce cas, les résultats relatifs aux épaisseurs optiques faibles se généralisent aisément : lorsque l'épaisseur optique est quelconque, les polarisations principales restent les mêmes; les indices principaux sont toujours donnés par la relation

$$
G=-i \frac{\omega l}{c}(N-1)
$$

(relation (I.7)), où $G$ est la matrice que nous avons étudiée en détail dans les paragraphes précédents; la seule différence est qu'il faut conserver la relation exacte $M_{\omega}=\exp \left[i \frac{\omega l}{c}(N-1)\right]$ sans développer l'exponentielle en se limitant au premier ordre.

Manuscrit reçu le 27 février 1967.

\section{BIBLIOGRAPHIE}

[1] Cohen-Tannoudji (C.) et Lalö̈ (F.), J. Physique, 1967, 28, 505.

[2] Cohen-Tannoudji (C.), Thèse, Paris, $1962 ; A n n$. Physique, 1962, 7, 423 et 469.

[3] Barrat (J. P.) et Cohen-Tannoudji (C.), J. Phys. Rad., 1961, 22, 329 ; J. Phys. Rad., 1961, 22, 443. Ces articles sont désignés par JP 1 et JP 2.

[4] NÉdÉlEc (O.), Thèse, Grenoble, 1966.

[5] Voir par exemple :

Messiah (A.), Mécanique Quantique, Dunod, Paris, 1960 , p. 485 et 924 .

FANO (U.) et RACAH (G.), Irreducible tensorial sets, Academic Press Inc., New York, 1959.

[6] Messiah (A.), op. cit., p. 920, formule (61).

[7] Manuer (J.) et Cohen-Tannoudji (C.), C. R. Acad. Sci. (Fr.), 1963, 257, 413.
[8] Brossei, (J.), Thèse, Paris, 1951; Ann. Physique, 1952, 7, 622.

Brossel (J.) et BitTé (F.), Phys. Rev., 1952, 86, 311.

[9] Corney (A.), Kibble (B. P.) et Serres (G. W.), Proc. Roy. Soc., A, 1966, 293, 70.

[10] Bouchiat (M. A.), Thèse, Paris, 1964.

[11] Bouchiat (M. A.), J. Physique, 1965, 26, 415.

[12] Bouchiat (M. A.) et Grossetî̂TE (F.), J. Physique, 1966, 27, 353.

[13] Dehmeli (H. G.), Phys. Rev., 1957, 105, 1924. Bel. (W. E.) et Bloom (A. L.), Phys. Rev., 1957, 107, 1559.

[14] Messiah (A.), op. cit., chap. XVII.

[15] Omont (A.), J. Physique, 1965, 26, 576. 